\title{
Sign-Selected Quadrupole Train
}

\author{
R. Bernstein et al \\ NuTev Collaboration \\ Fermi National Accelerator Laboratory \\ P.O. Box 500, Batavia, Illinois 60510
}

April 1994 


\section{Disclaimer}

This report was prepared as an account of work sponsored by an agency of the United States Government. Neither the United States Government nor any agency thereof, nor any of their employees, makes any warranty, express or implied, or assumes any legal liability or responsibility for the accuracy, completeness, or usefulness of any information, apparatus, product, or process disclosed, or represents that its use would not infringe privately owned rights. Reference herein to any specific commercial product, process, or service by trade name, trademark, manufacturer, or otherwise, does not necessarily constitute or imply its endorsement, recommendation, or favoring by the United States Government or any agency thereof. The views and opinions of authors expressed herein do not necessarily state or reflect those of the United States Government or any agency there of. 


\title{
들 Fermilab
}

\author{
Technical Memorandum \\ Sign-Selected Quadrupole Train
}

\author{
R. Bernstein et al. \\ NuTeV Collaboration \\ May 3, 1994
}

\begin{abstract}
The design of the Sign-Selected Quadrupole Train for E-815 (NuTeV) is set forth. The relevant physics requirements are explained. The optics of the beam are presented, along with an explanation of the proton dumping scheme. A discussion of rates and backgrounds follows, with special care given to backgrounds from scraping and obstructions. The relevant tolerances for beam construction are given and justified by simulations of the beamline. This leads to a discussion of the beam monitoring.
\end{abstract}




\section{Contents}

1 Physics Requirements and Design Goals $\quad 6$

1.1 Sign-Selection and Backgrounds ................. 7

1.2 Statistical Requirements . . . . . . . . . . . . . . . 7

2 Sign-Selected Quadrupole Train $\quad 9$

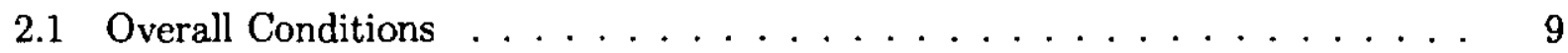

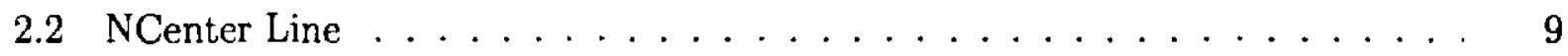

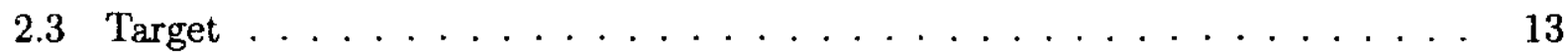

2.4 Sign-Selected Quadrupole Train Optics . . . . . . . . . . . . . . . 14

2.5 Optimization of the SSQT Tune . . . . . . . . . . . . . . . 19

2.6 Rates and Acceptances . . . . . . . . . . . . . . . . . 20

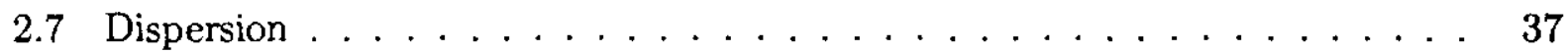

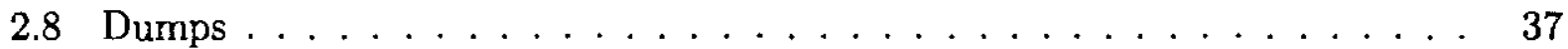

$\begin{array}{llr}3 & \text { Backgrounds } & 39\end{array}$

3.1 Beam Simulation Codes . . . . . . . . . . . . . . . 40

$3.2 \nu_{e}$ Production in the Beam ................... 40

$3.2 .1 \quad K^{ \pm} \rightarrow \pi e v_{e} \ldots \ldots \ldots \ldots \ldots$

$3.2 .2 \quad K_{L} \rightarrow \pi e \nu_{e} \ldots \ldots \ldots \ldots \ldots$

3.3 Production of $K_{L}$ in Dumps, Windows, Flanges, and Pipes . . . . . . . 44

3.3 .1 Production in Dumps ...................... 44

3.3.2 Production in Windows .................. 44

3.3.3 Production in Flanges and Pipes ............... 44

3.4 Wrong-Sign Backgrounds . . . . . . . . . . . . . . . . 45 


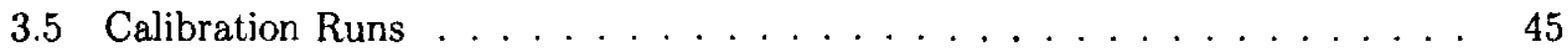

4 Beam Monitoring and Alignment $\quad 47$

4.1 Primary Beam Monitoring . . . . . . . . . . . . . . . . 47

4.2 Secondary Beam Monitoring . . . . . . . . . . . . . . . . 49

4.3 Alignment . . . . . . . . . . . . . . . . . . 49 


\section{List of Figures}

1 The CCFR detector, showing relevant characteristics. . . . . . . . 8

2 Layout of the Neutrino Area Beamlines. . . . . . . . . . . . 10

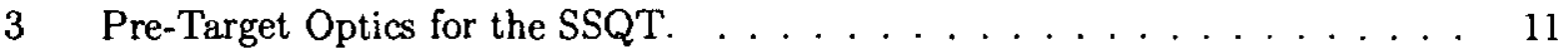

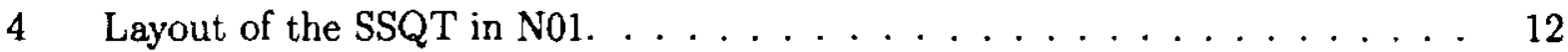

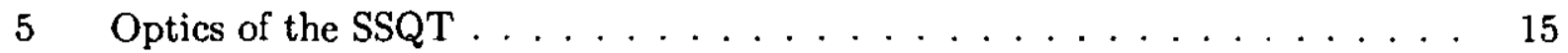

6 A schematic of the Sign-Selected Quadrupole Train in Neutrino Mode. Note the arrangement of the dumps, and that $900 \mathrm{GeV}$ protons will still hit the

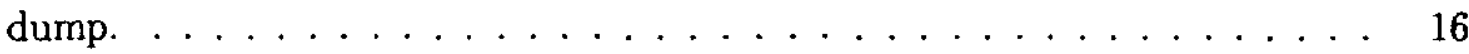

7 A schematic of the Sign-Selected Quadrupole Train in Antineutrino Mode. Note the first dump has been lowered, and that $900 \mathrm{GeV}$ protons will still hit the dump.

$8 \quad$ Interaction Rate $v s$. Field Strength for a $7.8 \mathrm{mrad}$ bend. . . . . . . . . . 23

9 Bend and Non-Bend Plane Angular Acceptance vs. Q1 field. . . . . . . . . 24

10 Angular Phase Space and Interaction Rate vs. Q1 Strength. . . . . . . . 25

11 Interaction Rates vs. Q3 Field for Various Beam Tunes. . . . . . . . . . . 26

12 Rate vs. Q4 Position for Standard Tune. . . . . . . . . . . . . . . 27

13 Rates vs. Q4 Position for Achromatic Tune. . . . . . . . . . . . 28

$14 p, \theta$ Acceptance of the train for sign-selected mesons in the bend (plots (a) and (c)) and non-bend (plots (b) and (d)) views. The normalization in the lego plots is arbitrary.

$15 \theta$ Acceptance of the train for neutrals $\left(K_{L}\right)$-independent of mode. The acceptance at a fixed $\theta$ is independent of momentum for neutrals.

16 Momentum Spectra of $\pi^{+}, K^{+}$mesons (1) produced at the target and (2) leaving the train, normalized per incident proton (in neutrino mode). The upper curve is the sum of the $\pi^{+}$and $K^{+}$rates, which, when integrated over angle, yields a sum of two exponentials.

17 Momentum Spectra of $\pi^{-}, K^{-}$mesons (1) produced at the target and (2) leaving the train, normalized per incident proton (in antineutrino mode). 
18 Momentum Spectra of $K_{L}$ mesons (1) produced at the target and (2) leaving the train, normalized per incident proton. . . . . . . . . . .

19 Momentum Spectrum of $\pi^{+}, K^{+}$Mesons, Weighted for Resultant Neutrino Interactions. The corresponding plot for $\bar{\nu}_{\mu}$ is similar in shape but is lower by the ratio of $\bar{\nu}_{\mu} / \nu_{\mu}$ rates. $\ldots \ldots \ldots \ldots \ldots \ldots \ldots \ldots \ldots \ldots \ldots$

20 Comparison of the Rate and Spectrum of the SSQT to the E-770 Quadrupole Triplet, for neutrinos and antineutrinos. Events satisfy a $50^{\prime \prime}$ radial cut.

21 Rates and Backgrounds for $\nu_{\mu}$ and $\bar{\nu}_{\mu}$ modes in the SSQT. All backgrounds have been included. The simulation has a radial cut of $50^{\prime \prime} . \ldots \ldots 36$

22 Impact Position of the Neutrinos on the Lab $\mathrm{E}$ detector. . . . . . . . .

23 Mean momentum as a function of position in the Lab $\mathrm{E}$ detector. Note the suppressed zero. . . . . . . . . . . . . . .

24 Comparison of Beam Simulation program to E-770 Data. The histogram is the data, and the crosses are the simulation. . . . . . . . . . . .

25 Relative Spectra of (1) $\nu_{\mu}$ from all charged current decays, and (2) $\nu_{e}$ from $K \rightarrow \pi e \nu_{e}$ for neutrino mode. . . . . . . . . . . . . . . . .

26 Overlap in $K^{ \pm}$momentum for $K^{ \pm} \rightarrow \mu \nu_{\mu}$ and $K^{ \pm} \rightarrow \pi e \nu_{e}$. The curve labeled $\nu_{\mu}$ is the momentum spectrum of $K^{+}$responsible for accepted $\nu_{\mu}$; the curve labeled $\nu_{e}$ is the momentum spectrum of $K^{+}$responsible for $\nu_{e}$. . . . . 43

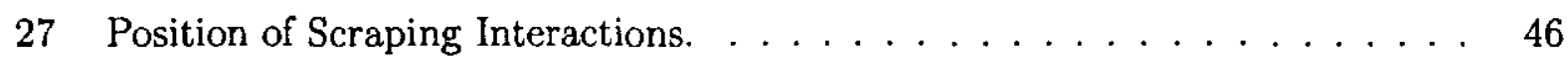

28 Effect of Beam Spot Size on the $\nu_{\mu}\left(K^{ \pm}\right)$spectrum. . . . . . . . . 48

29 Beam Profile at $\mathrm{NW} 2 \ldots \ldots \ldots \ldots \ldots \ldots \ldots \ldots \ldots$

30 Beam Profile at NW4. . . . . . . . . . . . . . . 51

31 Effect of a displacement of quadrupoles in the SSQT. The calculation is done for Q1 displaced downwards by 30 mils, Q2 upwards by 30 mils, and Q3 downwards by 30 mils. . . . . . . . . . . . . . . . . 


\section{List of Tables}

1 Names and Properties of Structures in the Neutrino Line. Locations are to the Nearest Foot, and precise pipe diameters are found in the appropriate engineering drawings. . . . . . . . . . . . .

2 Beam Sheet for the Sign-Selected Quadrupole Train. All needed magnets are available and this set of optics is compatible with pre-target requirements in N01. Magnetic Lengths are reported; the placement in $z$ includes spacing for

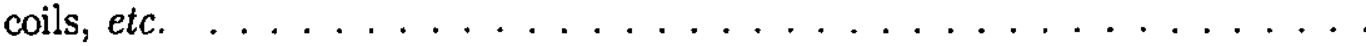

3 Charged Current Rates and Backgrounds in the SSQT. The rates assume $10^{13}$ POT at $800 \mathrm{GeV}$ and a $\nu_{\mu}$ impact point within $50^{\prime \prime}$ of the center. The full length of the detector is used. Scraping in beam pipes is included in the

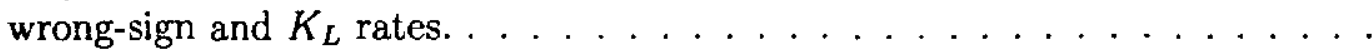

4 Statistical Power of E815, for a $1 \times 10^{18}$ POT run at $800 \mathrm{GeV}$, (split evenly between $\nu_{\mu}$ and $\bar{\nu}_{\mu}$ ) with the expected analysis cuts. The following analysis cuts have been applied: $E_{H}>20 \mathrm{GeV}$, radius $<40^{\prime \prime}$, and the vertex must be in the upstream $80 \%$ of the detector (to ensure longitudinal containment of the hadronic shower). These are representative of the cuts expected for the E815 analysis. . . . . . . . . . . . . . . . . . 


\section{Physics Requirements and Design Goals}

This report sets forth the design of a new Sign-Selected Quadrupole Train beam (SSQT). The beam has been designed to meet the needs of E-815, which will measure the electroweak parameters $\sin ^{2} \theta_{w}$ to \pm 0.003 and $\rho^{2}$ to \pm 0.01 . E815 will provide a factor of two improvement over the current best measurement of $\sin ^{2} \theta_{W}$ in DIS (E-770) and a factor of six in $\rho$ (from CDHS). $[1,2,3]$

The electroweak parameters will be extracted from the measurement of the ratios:[4]

$$
\begin{aligned}
& R_{\nu}=\frac{\sigma\left(\nu_{\mu}, N C\right)}{\sigma\left(\nu_{\mu}, C C\right)} \\
& R_{\bar{\nu}}=\frac{\sigma\left(\bar{\nu}_{\mu}, N C\right)}{\sigma\left(\bar{\nu}_{\mu}, C C\right)}
\end{aligned}
$$

The measurements require the separation of neutrino and antineutrino events. In charged current events, the sign of the outgoing muon is used to distinguish $\nu_{\mu}$ from $\bar{\nu}_{\mu}$. Neutral currents have no outgoing muon; hence, in order to separate neutral current $\nu_{\mu}$ from $\bar{\nu}_{\mu}$, the beam must provide the separation. The Quadrupole Triplet beam of E-744/E-770, which focuses both particles and anti-particles, will not suffice. The new beam must be sign-selected.

This two-equation system (Eq. 1,2) will enable E815 to extract the two unknowns $\rho$ and $\sin ^{2} \theta_{W}$. Sign-selection will also make it possible to perform the first measurement of the Paschos-Wolfenstein parameter

$$
R^{-}=\frac{\sigma\left(\nu_{\mu}, N C\right)-\sigma\left(\bar{\nu}_{\mu}, N C\right)}{\sigma\left(\nu_{\mu}, C C\right)-\sigma\left(\bar{\nu}_{\mu}, C C\right)}
$$

without knowledge of the absolute cross-sections. This is because dividing the numerator and denominator of $R^{-}$by $\sigma\left(\nu_{\mu}, C C\right)$ yields

$$
R^{-}=\frac{R_{\nu}-r R_{\bar{\nu}}}{1-r}
$$

where

$$
r=\frac{\sigma\left(\bar{\nu}_{\mu}, C C\right)}{\sigma\left(\nu_{\mu}, C C\right)}
$$

has already been measured in $\mathrm{E}-744 / \mathrm{E}-770 .^{1}$

\footnotetext{
${ }^{1} \mathrm{E}-744 / \mathrm{E}-770$ measured $\tau$ and the required structure functions. A full analysis is of course more complicated than this brief discussion indicates.
} 


\subsection{Sign-Selection and Backgrounds}

The sign-selection required to measure $R_{\nu}$ and $R_{\bar{\nu}}$ is achieved by adding vertical bends to a Quadrupole Train. At the same time, the vertical bends reduce the background from $K_{L} \rightarrow \pi e \nu_{e}$. In order to understand this and other sources of background we must describe the detector.

$\mathrm{NuTeV}$ will use the CCFR detector shown in Fig. 1. The "target" is 690 tons of iron interspersed with drift chambers and scintillation planes: one drift chamber every eight inches of $\mathrm{Fe}$, and a scintillation plane every four inches. A hadronic shower resulting from a neutrino interaction develops and dies out over 1-2 meters of iron. On this scale, an electromagnetic shower only covers $10-20 \mathrm{~cm} ; e^{-}$from charged-current $\nu_{e}$ are generally lost in the hadronic shower. Hence $\nu_{e}$ charged current interactions are effectively indistinguishable from $\nu_{\mu}$ neutral current interactions. ${ }^{2}$

Nearly all of the $\nu_{e}$ are from $K^{+} \rightarrow \pi^{\circ} e \nu_{e}$ decays. These are predicted through modeling of the beam; the level and spectrum of $K^{+}$production can be adequately constrained from the $K^{+} \rightarrow \mu \nu_{\mu}$ decays which provide the high energy end of the $\nu_{\mu}$ data.

There is no equivalent way to predict the $\nu_{\mathrm{e}}$ rate from $K_{L}$ decay; the $K_{L} \rightarrow \pi \mu \nu_{\mu}$ rate is so small that it is lost in the larger $K^{ \pm}$signal. The absolute level of $K_{L}$ production is not known to better than $20 \%$. This error in the $K_{L}$ rate produced an error of $\approx \pm 0.003$ on the E-770 extraction of $\sin ^{2} \theta_{W}$. Our goal is to reduce the $K_{L}$ contribution to where its uncertainty is a negligible contribution to the total error. Our final design will reduce the $K_{L}$ contribution relative to $\mathrm{E}-770$ by an order-of-magnitude, yielding an error of no more than \pm 0.0003 , one-tenth the final error.

\subsection{Statistical Requirements}

The $\mathrm{E}-770$ run (E-744 was not used in the $\sin ^{2} \theta_{W}$ analysis) provided $250 \mathrm{~K}$ neutral current events from $5 \times 10^{17}$ protons-on-target and a final statistical error of \pm 0.0025 . We must have a similar or better statistical error in E815. An extraction of $\sin ^{2} \theta_{W}$ using $R^{-}$and the Paschos-Wolfenstein technique has a larger statistical error than the corresponding Llewellyn Smith analysis, because $R^{-}$is formed from the difference of neutrino and antineutrino rates; the statistical error is dominated by the number of anti-neutrino neutral current events. ${ }^{3}$

\footnotetext{
${ }^{2}$ Attempts have been made to perform the separation using the energy deposit as a function of length into the shower; the technique is not sufficiently accurate to be of use. Tracking in this environment is not feasible.

${ }^{3}$ A global anal ysis of the errors shows that equal neutrino and antineutrino running optimizes the error. It is also true that in a two-parameter fit, with properly correlated errors, there is no difference in the extracted parameters or their final errors between the Paschos-Wolfenstein and Lllewellyn Smith analyses.
} 


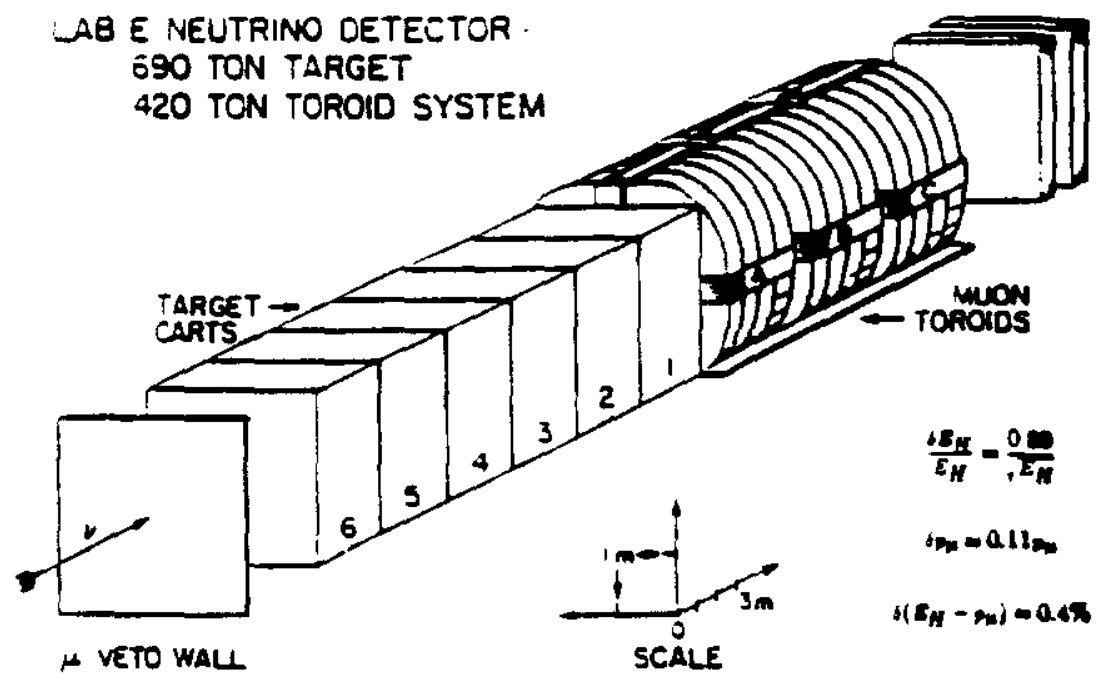

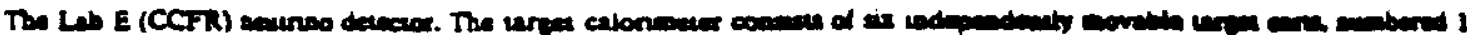

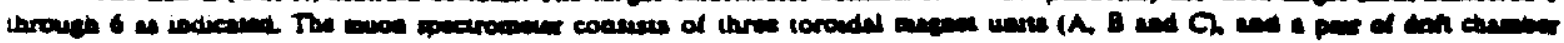

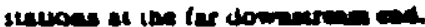

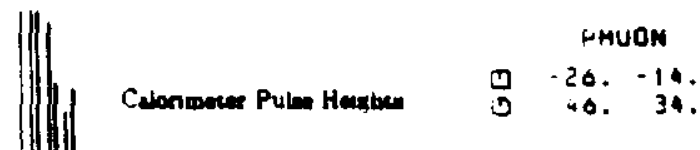

ETOT 387.9 EHAO 315.6

$1 \quad$ U

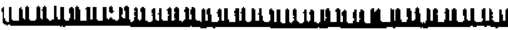

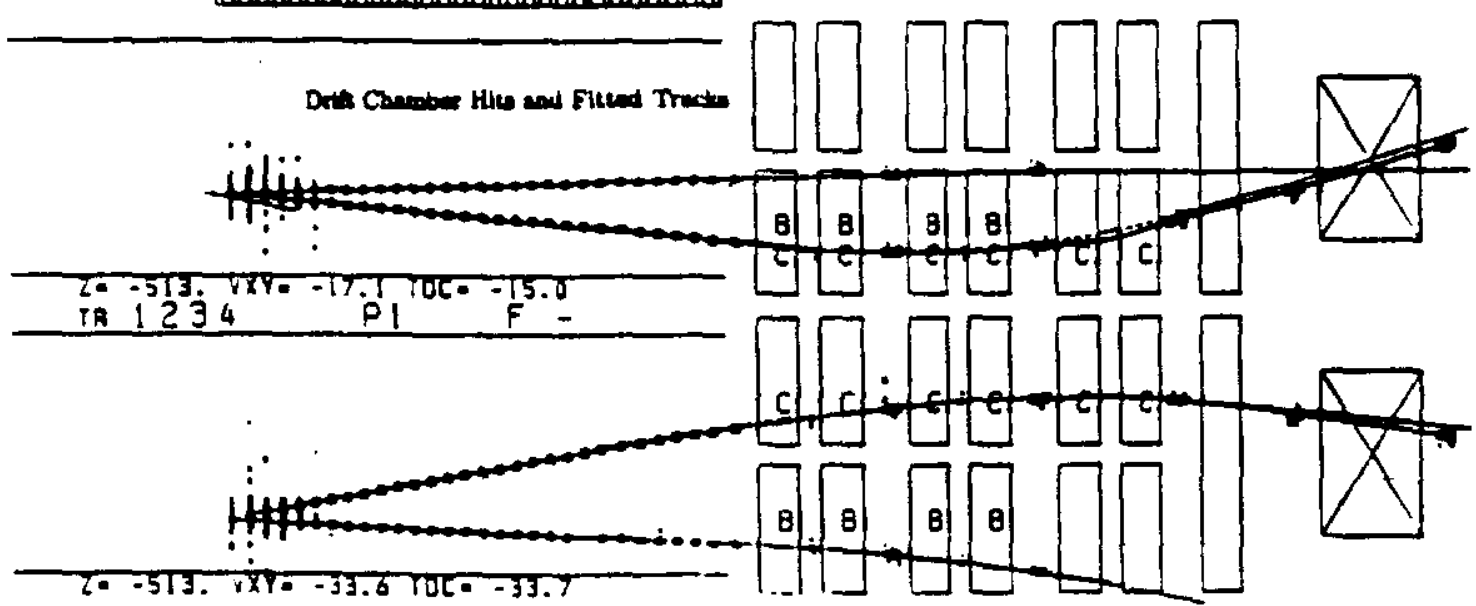

Figure 1: The CCFR detector, showing relevant characteristics. 
A dichromatic beam (such as the one used in E-616/701 or proposed in TM-841) would provide excellent neutrino/antineutrino separation and minimum $\nu_{e}$ background. However, since the beam is momentum selected the experiment would be systematically clean but severely limited statistically. We have chosen a new alternative: a Sign-Selected Quadrupole Train. It will cleanly separate $\nu_{\mu}$ from $\bar{\nu}_{\mu}$ and eliminate $K_{L}$ decays as a significant source of error.

\section{Sign-Selected Quadrupole Train}

\subsection{Overall Conditions}

The Sign-Selected Quadrupole Train will reside in N01. The entire train will sit on a "railroad" so that if needed, the train can be pulled out and moved to the Target Service Building for repairs.[9]

The experiment will take between three and five pings (or more properly, fast resonant extractions) separated by approximately $1 \mathrm{sec}$. Each ping will have intensities of the order of $3-5 \times 10^{12}$ POT. We plan for all pings to be at the end of the slow spill to avoid potential disruption to slow-spill users. We may choose to run with only one ping in anti-neutrino mode.

\section{$2.2 \quad$ NCenter Line}

A variety of naming schemes have been used in the Neutrino Area, and all are still in use. To avoid confusion and refresh the reader's memory, we summarize below the names, $z$ locations, and relevant properties of the NC line in Table 1. Fig. 2 shows each of the lines in the Neutrino Area. The pre-target optics is shown in Fig. 3, followed by a layout of the train in N01 in Fig. 4.

The vacuum system of E-744/E-770 has been changed to lessen backgrounds from scraping and obstructions in the following manner:

1. The vacuum will run from within the train after Q2 (NC1Q4 in Ref. [9]) until the end of the decay pipe at NW4.

2. A $12^{\prime \prime}$ diameter vacuum pipe will start at the end of the SSQT and run continuously to the end of the Target Tube, eliminating the old window at the entrance to the Target Tube. 


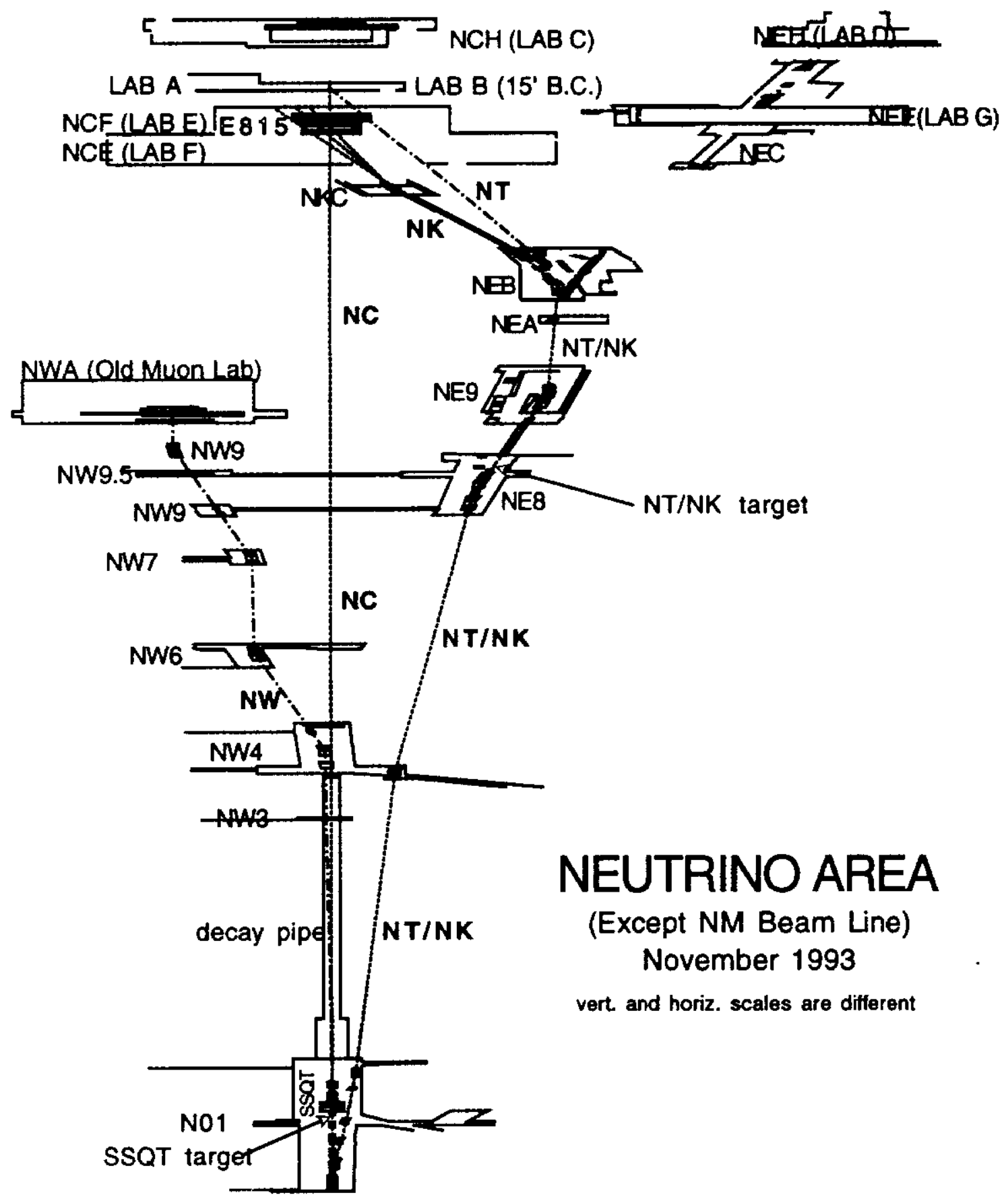

Figure 2: Layout of the Neutrino Area Beamlines. 


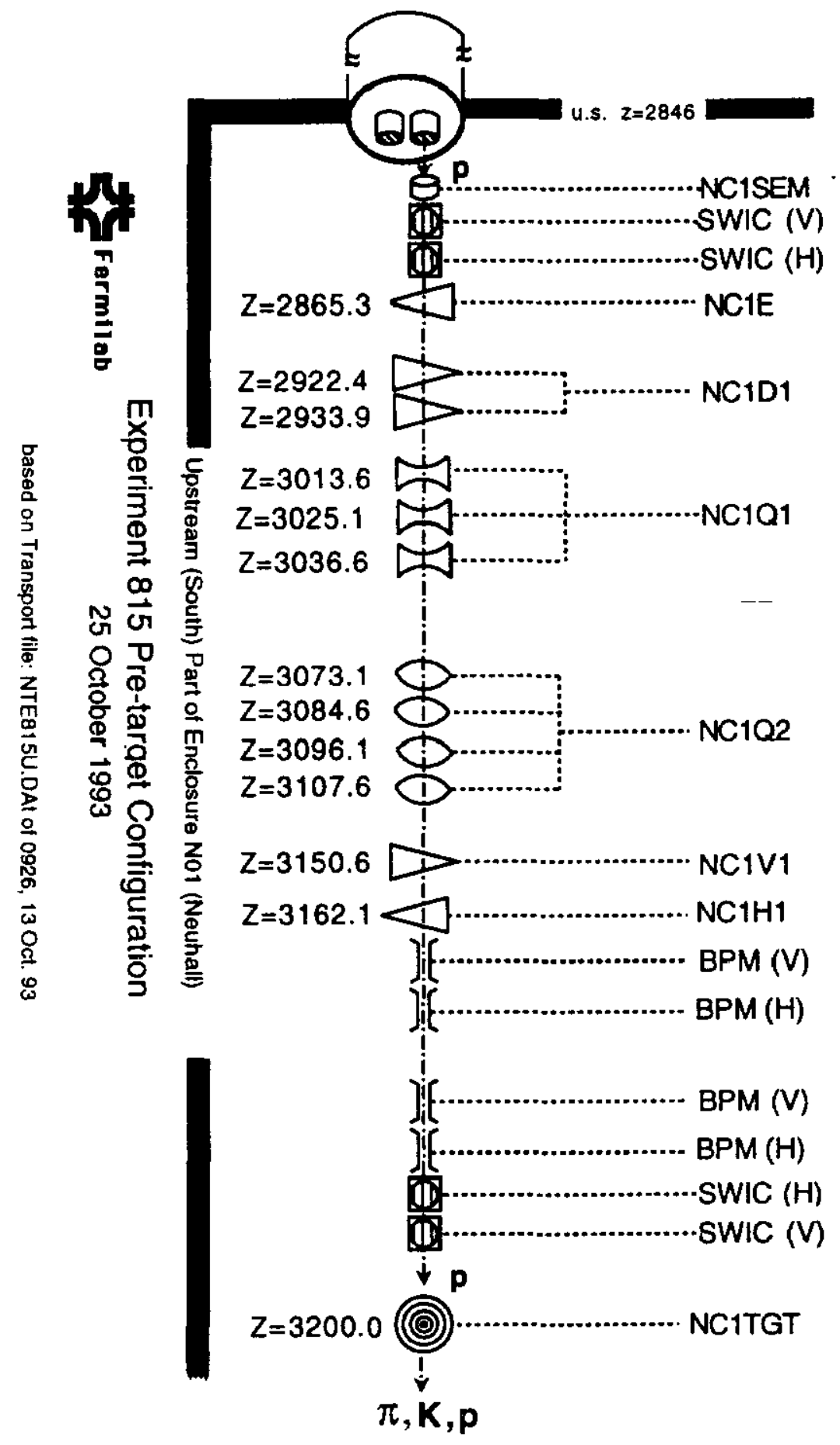

Figure 3: Pre-Target Optics for the SSQT. 


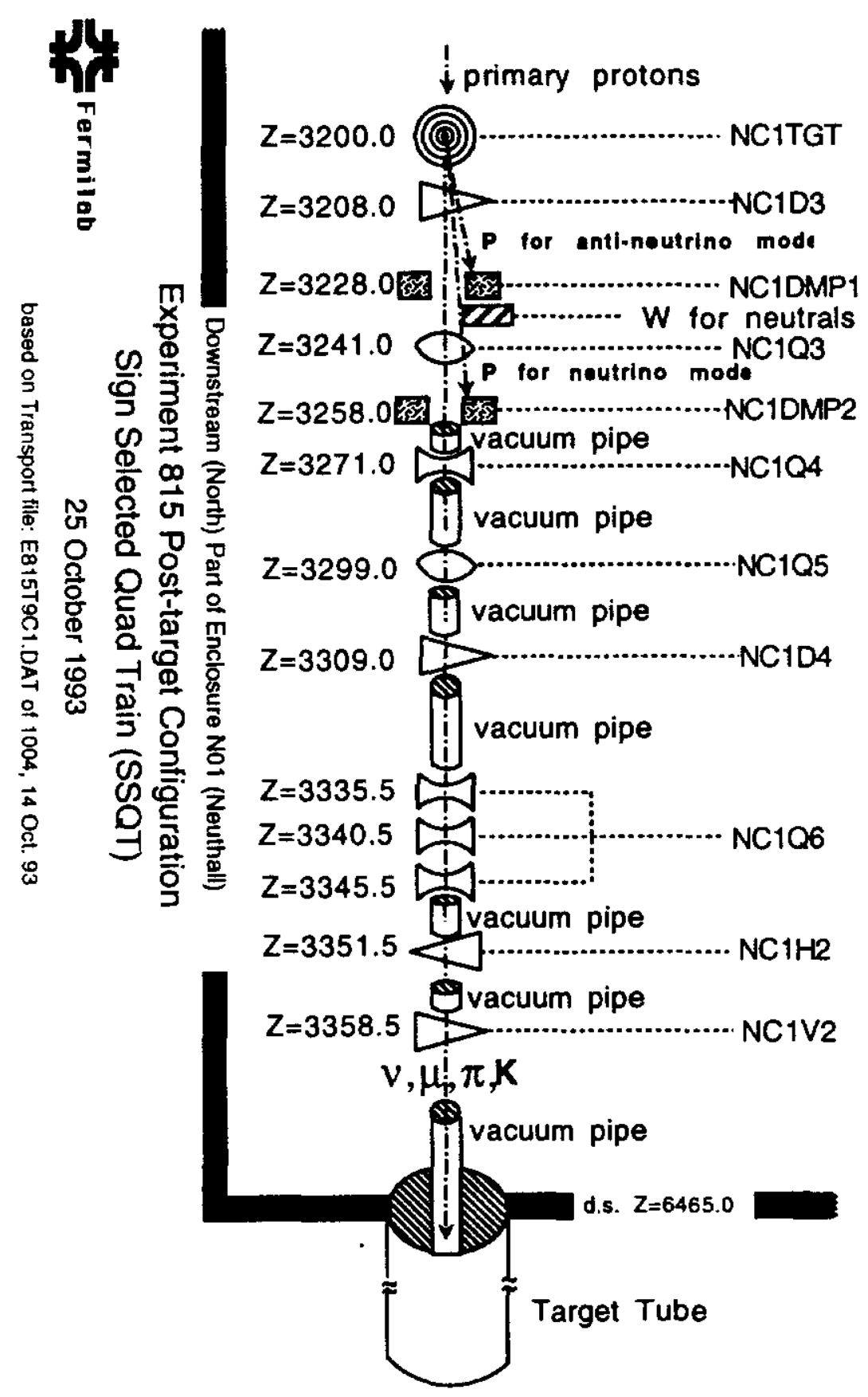

Figure 4: Layout of the SSQT in N01. 


\begin{tabular}{lccll} 
Section & Begin(DUSAF, ft) & End & Diameter & Comments \\
\hline South End Extension & 2846 & 3146 & Beam Hall & \\
N01 & 3146 & 3457 & Beam Hall & SSQT Target at 3200 \\
Target Tube & 3460 & 3760 & $17^{\prime \prime}$ l.D. Vac Pipe & Surrounded by 6ft outer Tube \\
Decay Pipe & 3770 & 4816 & $36^{\prime \prime}$ I.D. Vac Pipe & \\
Expansion Port(NW2) & 4093 & 4103 & $36^{\prime \prime}$ & Secondary Monitoring \\
Target Manhole(NW3) & 4600 & 4610 & $36^{\prime \prime}$ & \\
E100(NW4) & 4796 & 4817 & $6^{\prime}$ & Dump Remaining Particles \\
NuTeV Detector(Lab E) & 7848 & 7908 & $10^{\prime}$ & Detector
\end{tabular}

Table 1: Names and Properties of Structures in the Neutrino Line. Locations are to the Nearest Foot, and precise pipe diameters are found in the appropriate engineering drawings.

3. The old window and flange arrangement at the Target Tube/Decay Pipe interface will be replaced with continuous vacuum pipe or a thin window. The existing system had an $8^{\prime \prime}$ diameter window with a thick $\mathrm{Al}$ flange, which would obstruct the E815 mesons. The new expansion piece will minimize the material in the region.

4. The windows at NW2 will be of similar construction: Ti or Kevlar/Mylar. Currently they are much thicker $\mathrm{Al}$ windows.

5. The windows at NW3 will be replaced with vacuum pipe.

\section{$2.3 \quad$ Target}

A low- $Z$ material is the most efficient for producing neutrinos. Beryllium oxide, used in E-744/E-770 and prior runs, has both good thermal and structural properties. The E-815 target will be a modified version of the target used in the 1987 Quad Triplet Train.

In that run, the target moved in and out of the beam several times every spill (the "dancing target"). During most of the flattop there was slow spill for NW and the E$744 / \mathrm{E}-770$ target, located along $\mathrm{NC}$, was out. Whenever there was fast spill (pings) the target was moved in and NC took data. For the E-815 run NC and NW share E-815's target. This allows several changes to be made:

1. The aluminum in the cooling block is replaced with copper. With aluminum there is always the possibility of forming aluminum oxide (a fairly good insulator that will impede the heat flow away from the $\mathrm{BeO}$.)

2. The cooling lines are better positioned radially and have a larger diameter. In addition, all cooling lines are in parallel transverse to the beam direction. 
3. Two identical $12^{\prime \prime}$ long, $1^{\prime \prime}$ diameter $\mathrm{BeO}$ targets are provided.

4. The entire upper section ( $\mathrm{BeO}$ plus copper cooling block) can easily be removed from its baseplate for disassembly or replacement.

5. The target is mounted on a movable drive that has three positions:

- Beam centered on either of the two targets.

- Target out.

The two targets are in side-by-side stations; one is a backup to the other. At periodic times during the run, one target can be switched for the other to check the integrity of the standard target.

A detailed analysis shows this water-cooled $\mathrm{BeO}$ target will survive $1 \mathrm{E} 13$ protons per ping. [9] For 3E13, three or more pings are required. ${ }^{4}$

\subsection{Sign-Selected Quadrupole Train Optics}

A layout of the pre-target optics is shown in Fig. 3. The pre-target magnets will deliver the $800 \mathrm{GeV}$ primary beam ${ }^{5}$ from the Tevatron at an upward angle of $7.8 \mathrm{mrad}$ to the target, and provide a spot with $\sigma_{x} \approx 0.7 \mathrm{~mm}$ and $\sigma_{y} \approx 3 \mathrm{~mm}$. [9] The target is located at DUSAF $z=3200^{\prime}, 1417$ meters upstream of the upstream end of the Lab E detector. ${ }^{6}$

A picture of the optics of the SSQT is illustrated in Fig. 5; an accompanying beamsheet is given in Table 2.

Figs. 6 and 7 shows a schematic of the beamline with a central ray at $250 \mathrm{GeV}$ and the trajectories of $800 \mathrm{GeV}$ protons in neutrino and antineutrino running. There will be only two changes in switching from neutrino to antineutrino mode. We will first reverse the polarity of all magnets and then move the edges of both dumps appropriately.

After the protons strike the target, the resultant secondaries and remaining protons are bent through a modified B-2 (10') which bends $250 \mathrm{GeV}$ secondaries through $6.2 \mathrm{mrad}$. This is the bend providing the sign-selection: the selected polarity is bent toward Lab $\mathrm{E}$, the undesired polarity is bent away, and neutrals are unaffected and travel forward. Increasing the bend would lower both the background and the rate. The final choices optimize the signal/background ratio while also ensuring adequate statistics.

\footnotetext{
${ }^{4}$ Live-time requirements of the DAQ require a greater number of pings in neutrino mode.

${ }^{5}$ The train and optics will work at $900 \mathrm{GeV}^{\prime}$ as well, with a $20 \%$ increase in rate.

${ }^{6}$ In this discussion we have ignored the Earth curvature corrections, which will tilt the beamline by $\approx 0.08$ mrad with respect to the NCenter line. The corrections have been taken into account in engineering details.
} 

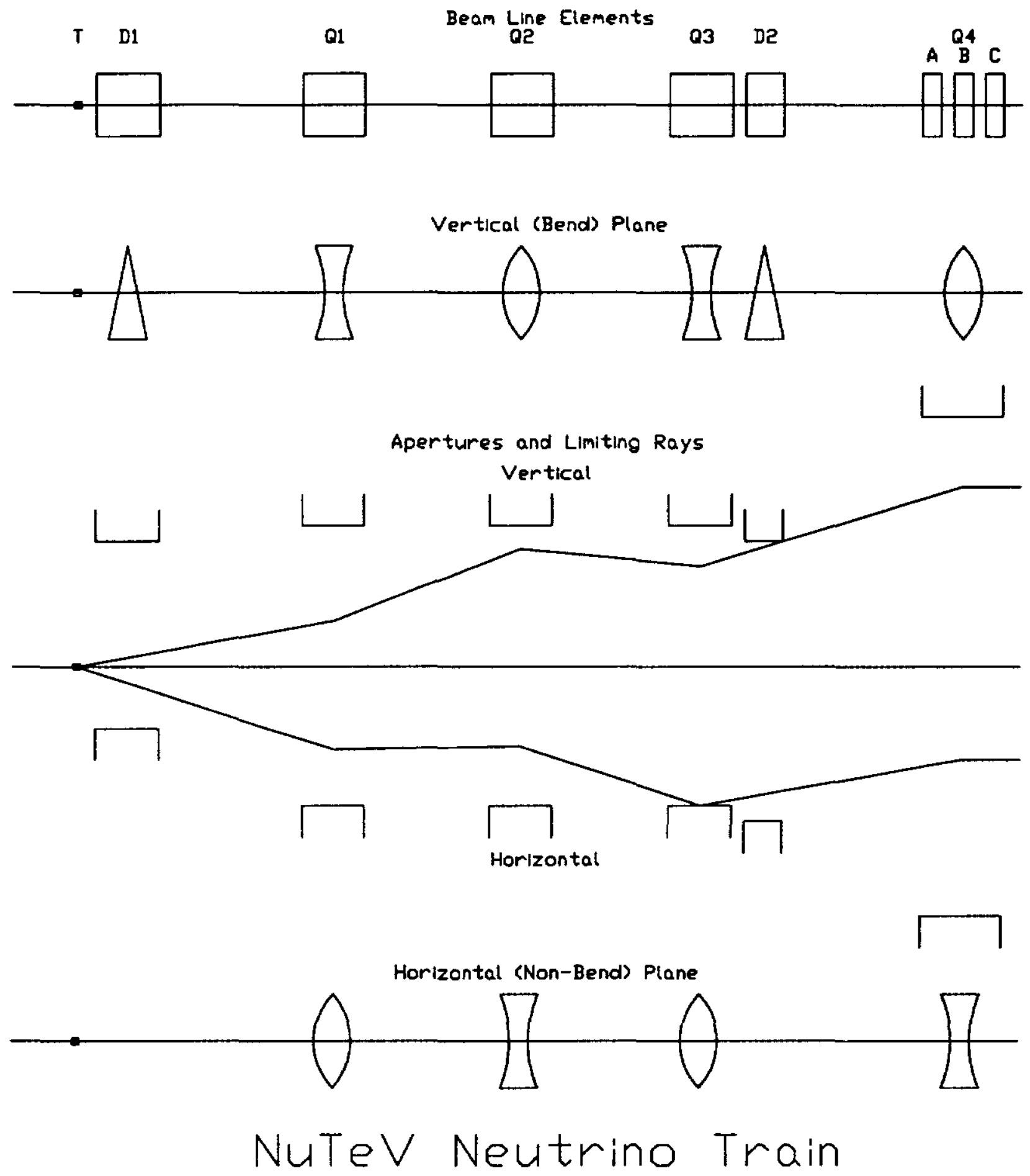

Figure 5: Optics of the SSQT 
Sign Selected Quad Train

(elevation view, horiz. and vert. scales different)

Neutrino Mode

26 Oct. 1993

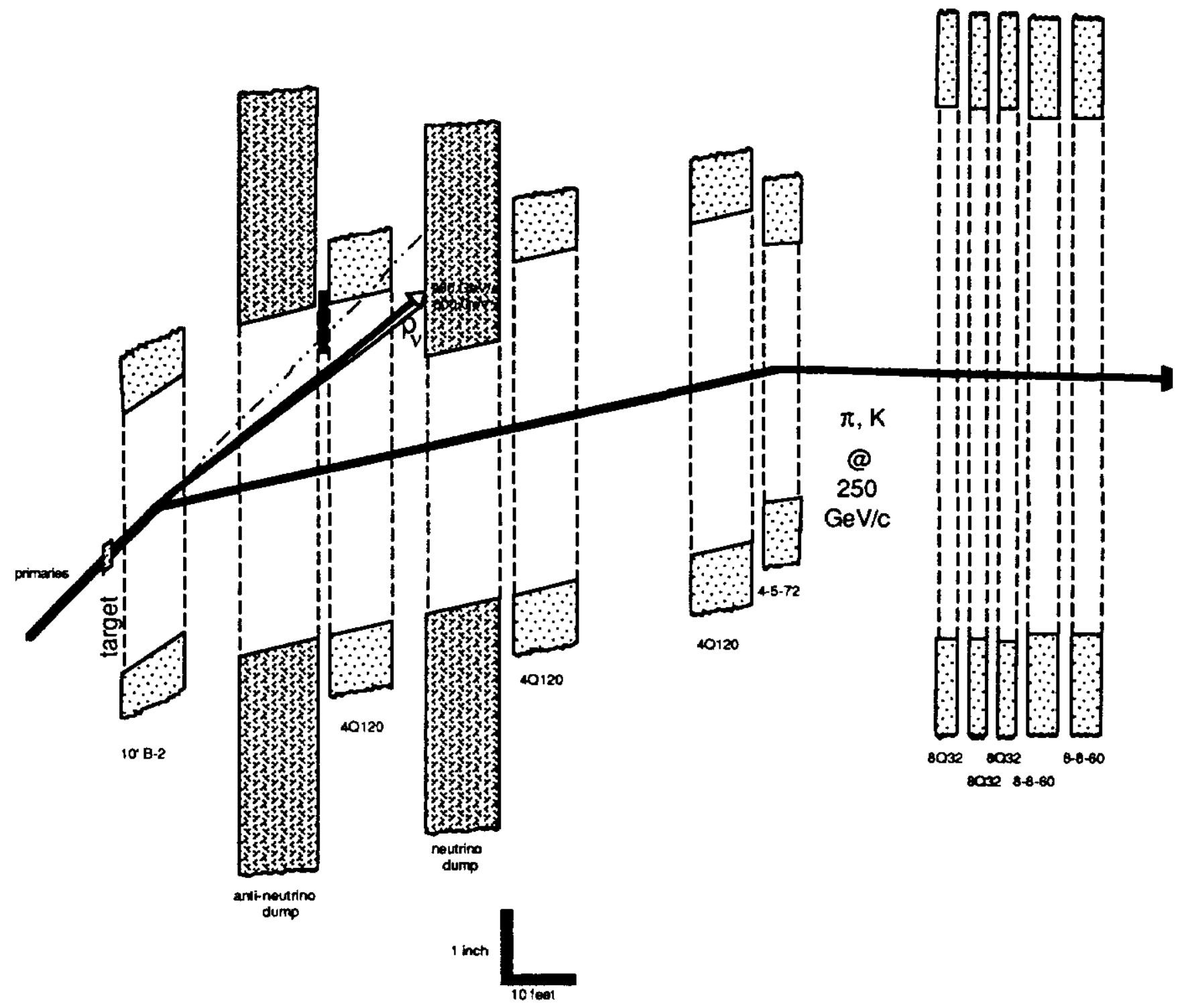

Figure 6: A schematic of the Sign-Selected Quadrupole Train in Neutrino Mode. Note the arrangement of the dumps, and that $900 \mathrm{GeV}$ protons will still hit the dump. 
Sign Selected Quad Train

(elevation view, horiz. and vert. scales different)

Anti-neutrino Mode

26 Oct. 1993

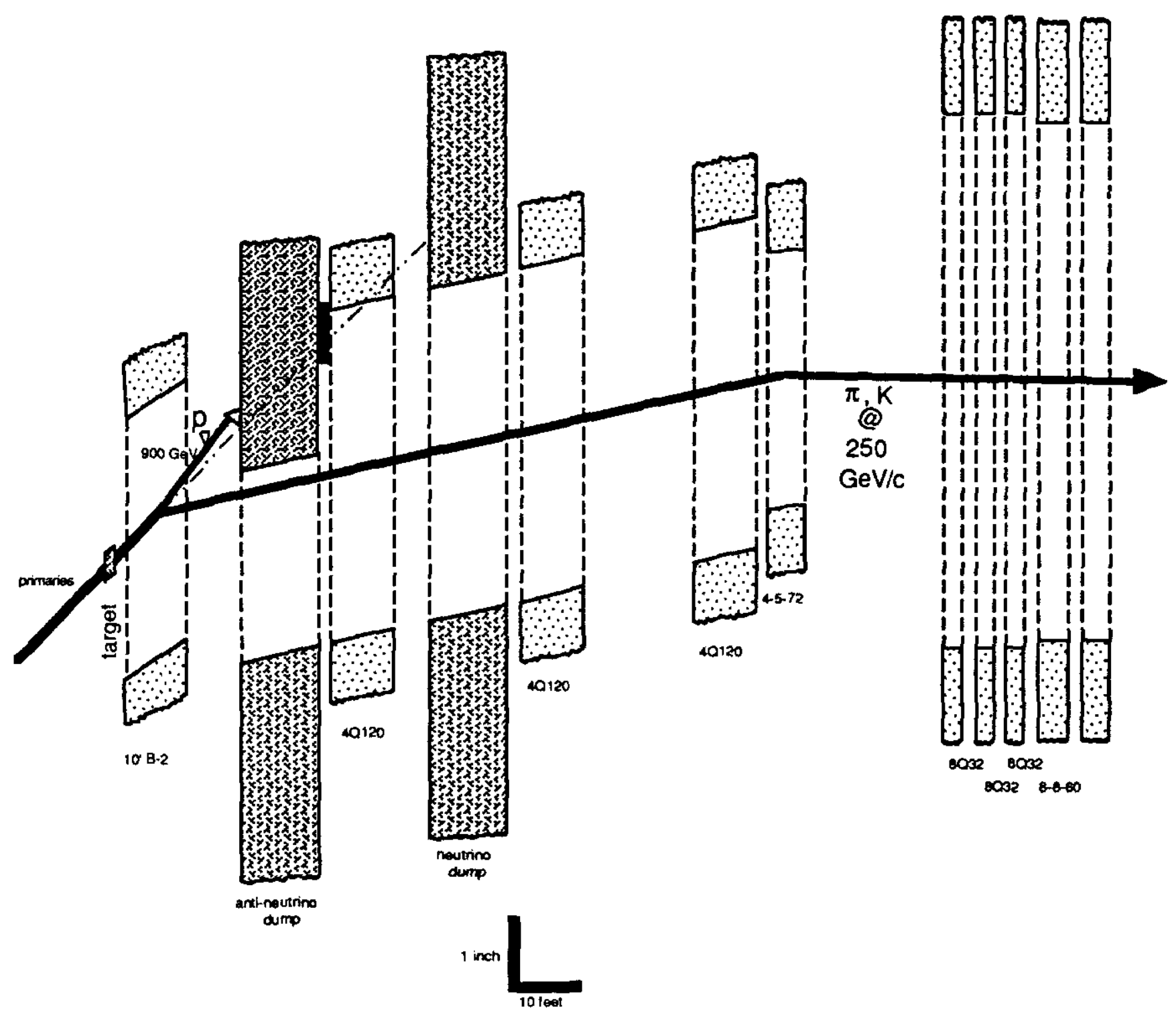

Figure 7: A schematic of the Sign-Selected Quadrupole Train in Antineutrino Mode. Note the first dump has been lowered, and that $900 \mathrm{GeV}$ protons will still hit the dump. 


\begin{tabular}{|l|l|l|l|c|c|c|c|}
\hline & Device & Name & RD Name & $\mathrm{B}(\mathrm{kg}, \mathrm{kg} / \mathrm{in})$ & $z_{\text {center }}(m)$ & Length $(\mathrm{ft})$ & $y_{\text {center }}(\mathrm{m})$ \\
\hline 1 & Target & & & 0.0 & 0 & $1 \lambda_{0}$ & -0.068 \\
2 & Dipole (modified B2) & D1 & NCID3 & +17.0 & 2.4 & 10 & -0.049 \\
3 & $\bar{\nu}_{\mu}$ Dump & & 0. & 8.5 & 12 & -0.040 \\
4 & Quad 4Q120 & Q1 & NC1Q3 & -5.5 & 12.5 & 10 & -0.033 \\
5 & $\nu_{\mu}$ Dump & & & 0.0 & 17.7 & 12 & -0.025 \\
6 & Quad 4Q120 & Q2 & NC1Q4 & +5.5 & 21.6 & 10 & -0.019 \\
7 & Quad 4Q120 & Q3 & NC1Q5 & -4.97 & 30.2 & 10 & -0.005 \\
8 & Dipole(4-5-72) & D2 & NC1D4 & 8.00 & 33.2 & 6 & 0.0 \\
9 & Quad 8Q32 & Q4A & NC1Q6-1 & 2.65 & 41.3 & 3 & 0.0 \\
10 & Quad 8Q32 & Q4B & NC1Q6-2 & 2.65 & 42.8 & 3 & 0.0 \\
11 & Quad 8Q32 & Q4C & NC1Q6-3 & 2.65 & 44.3 & 3 & 0.0 \\
12 & V.Trim (8-8-60) & D3 & NC1D5 & 0. & 50.0 & 10 & 0.0 \\
13 & H.Trim (8-8-60) & D4 & NC1D6 & 0. & 56.0 & 10 & 0.0 \\
\hline
\end{tabular}

Table 2: Beam Sheet for the Sign-Selected Quadrupole Train. All needed magnets are available and this set of optics is compatible with pre-target requirements in N01. Magnetic Lengths are reported; the placement in $z$ includes spacing for coils, etc.

The non-interacting protons are also bent by the dipole. Since the polarity of the dipole reverses between neutrino and antineutrino modes, the outgoing trajectory of the protons changes as well. There is then one dump for each trajectory: (1) when negative secondaries are selected (antineutrino mode), the protons are bent upwards (away from the central ray) and dump in the first dump, and (2) when positives are chosen (neutrino mode) the protons are bent downward and strike the second dump. We defer further discussion of the dumps until Sec. 2.8 .

The quadrupoles have two functions:

- The first two quadrupoles (Q1 and Q2) capture the beam. They are run at maximum strength to maximize the captured phase space.

- The second group (Q3, Q4A, Q4B, and $\mathrm{Q} 4 \mathrm{C}$ ) direct the mesons essentially parallel to the NC Line toward Lab E.

A second small bend is placed between Q3 and the Q4 set. This bend accomplishes three functions:

1. It increases the overall bend angle of the beam further enhancing the sign-selection. 
2. It sweeps the low energy particles produced by scraping along the magnet apertures upstream away from the detector.

3. By redirecting the charged particle channel, it allows the neutral particle channel determined by upstream apertures to head away from the center of the detector, thus reducing our $K_{L}$ background uncertainty.

The final set of three quadrupoles, in conjunction with Q3 reform the captured beam into a quasi-parallel, quasi-achromatic beam. The parallelness minimizes scraping in pipes and flanges which would otherwise be a significant background. Because the distance between Q4 and the detector is large compared to the distance from the target to Q1, the magnification of the target spot size at the detector is large. With this tune, the final magnifications at the detector are 100 in the non-bend plane and 33 in the bend plane.

\subsection{Optimization of the SSQT Tune}

Various studies have been made to understand the tune characteristics of the SSQT. When choosing a tune, one must consider a number of competing factors: the interaction rates in the detector, scraping backgrounds, $K_{L}$ backgrounds, and our ability to unfold the production spectrum from the detected interactions. An empirical "best" tune has been used for the studies in this memo. While this "best" tune is not the best in any one sense, it is good in all senses.

The empirical "best" tune consists of running Q1 and Q2 at their maximum value and using Q3 and Q4 to direct $225 \mathrm{GeV} / \mathrm{c}$ particles parallel to the $\mathrm{NC}$ beamline in the non-bend plane and to focus them at the NW4 dump in the bend plane. As can be seen in Fig. 8, the interaction rate is maximized if the bend magnets are set slightly higher than the quad train. The amount of this effect is production model dependent. Historically, we have chosen the dipoles to bend $250 \mathrm{GeV} / \mathrm{c}$ particles down the beam axis because the backgrounds are less than at a dipole setting of $225 \mathrm{GeV} / \mathrm{c}$.

The angular acceptances in the bend and non-bend plane as a function of the Q1 field are illustrated in Figs. 9 and 10. Fig. 9 shows that the non-bend plane acceptance increases rapidly as the field in Q1 increases. At the same time, the acceptance in the bend plane decreases slowly. Fig 10 shows that the phase space acceptance, the product of the $x$ and $y$ plane acceptance, increases with Q1 field. Also shown in Fig. 10 is the NUADA estimate for the number of neutrino interactions in the CCFR detector for $10^{13}$ protons on target. The number of events does not increase as fast as phase space. Increasing the field in Q1 gains phase space at large angles and loses it at smaller angles but since the particle flux decreases with angle, running Q1 and Q2 at their maximum values maximizes both the captured phase space and the number of detected events. 
The currents in Q3 and Q4 determine the transport of the particles downstream of the SSQT. Fig. 11 shows the number of NUADA events for two different tunes: one which focuses the beam at the NW4 dump in the bend plane (BP), and one which makes the beam parallel in the non-bend plane (NBP). The intersection of the two curves is the point of the "best" tune. Note that you can find tunes that give higher interaction rates than the "best" tune, i.e. $4.5 \mathrm{~kg} / \mathrm{in}$ for Q3 in the berm focused tune. This tune point is actually defocusing the beam in the non-bend plane. How can this be? With the magnet string in the present design, D2 (a 4-5-72) is the limiting aperture for the non-bend plane. By reducing the current in Q3, we allow more particles through D2 and, thus, increase our event rate. If we eliminate this aperturing by using a large gap magnet for D2 (such as a 6-6-120), we can increase our expected interaction rate by $8 \%$. This is illustrated by the third curve in Fig. 11. Again, you can find tunes that are slightly higher, but now, with the removal of the D2 aperture, we are on a large, rather flat plateau. We have found points that are up to $3 \%$ higher interaction rates, but those points have lower average energies and are less desirable for that reason.

In addition to changes in the magnetic field strengths, we can also vary the positions of the train magnets. Figs. 12 and 13 show the interacting neutrino rate as a function of Q4 position for two different tunes: the standard tune which is parallel in the non-bend plane and focused at the NW4 dump in the bend plane (Fig. 12), and parallel in the non-bend plane and with no momentum dispersion at the detector (Fig. 13). As can be seen from these from these two plots, Q4 is well positioned for the large aperture D2, but should be moved downstream for the smaller magnet.

\subsection{Rates and Acceptances}

The raw rates for this configuration are given in Table 3; Table 4 gives the numbers of events we expect after the E-770 analysis cuts.

The acceptance of the train is shown in Fig. 14 for both sign-selected and Fig. 15 for neutrals. The plots show the fraction produced versus those accepted in $p$ and $\theta$, where $\theta=0$ is along the initial proton direction. Neutrino and anti-neutrino acceptances are the same in their respective modes.

Another way to look at the acceptance is shown in Fig. 16-18. Fig. 16 shows the momentum spectrum for $\pi^{+}$and $K^{+}$mesons produced at the target and after passing through the train for neutrino mode, and for antineutrino mode in Fig. 17; the acceptance for $K_{L}$ is shown in Fig. 18. Finally, we may study the "useful" spectrum. Low energy mesons do little for the rate since the neutrino cross-section rises linearly with energy. Figs. 19 shows the spectrum of hadrons, weighted for the number of resultant neutrino interactions.

Spectral comparisons to the E-770 QT are shown in Fig. 20 for neutrinos and antineutrinos. We see that the SSQT rates are $50-60 \%$ of the QT rates per proton; however, since half 


\begin{tabular}{|c|c|c|c|}
\hline$\nu_{\mu}$ Mode & Type & Number $/ 10^{13}$ POT & $<E_{\nu}>(\mathrm{GeV})$ \\
\hline & $\bar{\nu}_{\mu}$ & .03 & 58 \\
\hline & & 18.6 & 118 \\
\hline & $\nu_{e}\left(K_{L}\right)$ & $1.1 \times 10^{-2}$ & 45 \\
\hline $\bar{\nu}_{\mu}$ Mode & Type & Number $/ 10^{13}$ POT & $<E_{\bar{\nu}}>(\mathrm{GeV})$ \\
\hline & $\bar{\nu}_{\mu}$ & 3.6 & 99 \\
\hline & $\nu_{\mu}$ & .009 & 39 \\
\hline & $\nu_{e}\left(K_{L}\right)$ & $1.1 \times 10^{-2}$ & 45 \\
\hline
\end{tabular}

Table 3: Charged Current Rates and Backgrounds in the SSQT. The rates assume $10^{13}$ POT at $800 \mathrm{GeV}$ and a $\nu_{\mu}$ impact point within $50^{\prime \prime}$ of the center. The full length of the detector is used. Scraping in beam pipes is included in the wrong-sign and $K_{L}$ rates.

\begin{tabular}{ccc}
\multicolumn{3}{c}{$\mathrm{E} 815$} \\
& $\mathrm{NC}$ & $\mathrm{CC}$ \\
\hline$\nu_{\mu}$ & $113 \mathrm{~K}$ & $362 \mathrm{~K}$ \\
$\bar{\nu}_{\mu}$ & $18 \mathrm{~K}$ & $48 \mathrm{~K}$
\end{tabular}

Table 4: Statistical Power of E815, for a $1 \times 10^{18}$ POT run at $800 \mathrm{GeV}$, (split evenly between $\nu_{\mu}$ and $\bar{\nu}_{\mu}$ ) with the expected analysis cuts. The following analysis cuts have been applied: $E_{H}>20 \mathrm{GeV}$, radius $<40^{\prime \prime}$, and the vertex must be in the upstream $80 \%$ of the detector (to ensure longitudinal containment of the hadronic shower). These are representative of the cuts expected for the E815 analysis. 
the run will be in neutrino mode and half in anti-neutrino, the absolute number of events will suffer an additional factor of two loss. The reason for the high energy loss is straightforward: high-energy parent mesons are not bent into the acceptance and tend to strike one of the dumps.

The most important plots are, of course, the relative rates and backgrounds. We give these for each of neutrino and antineutrino modes in Fig. 21. Approximately $80 \%$ of the wrong-sign background comes from scraping. The largest source of scraping is the decay pipe. The remaining $20 \%$ comes from mesons which decay within the train or from mesons produced at high $p_{\perp}$ which escape the train. 


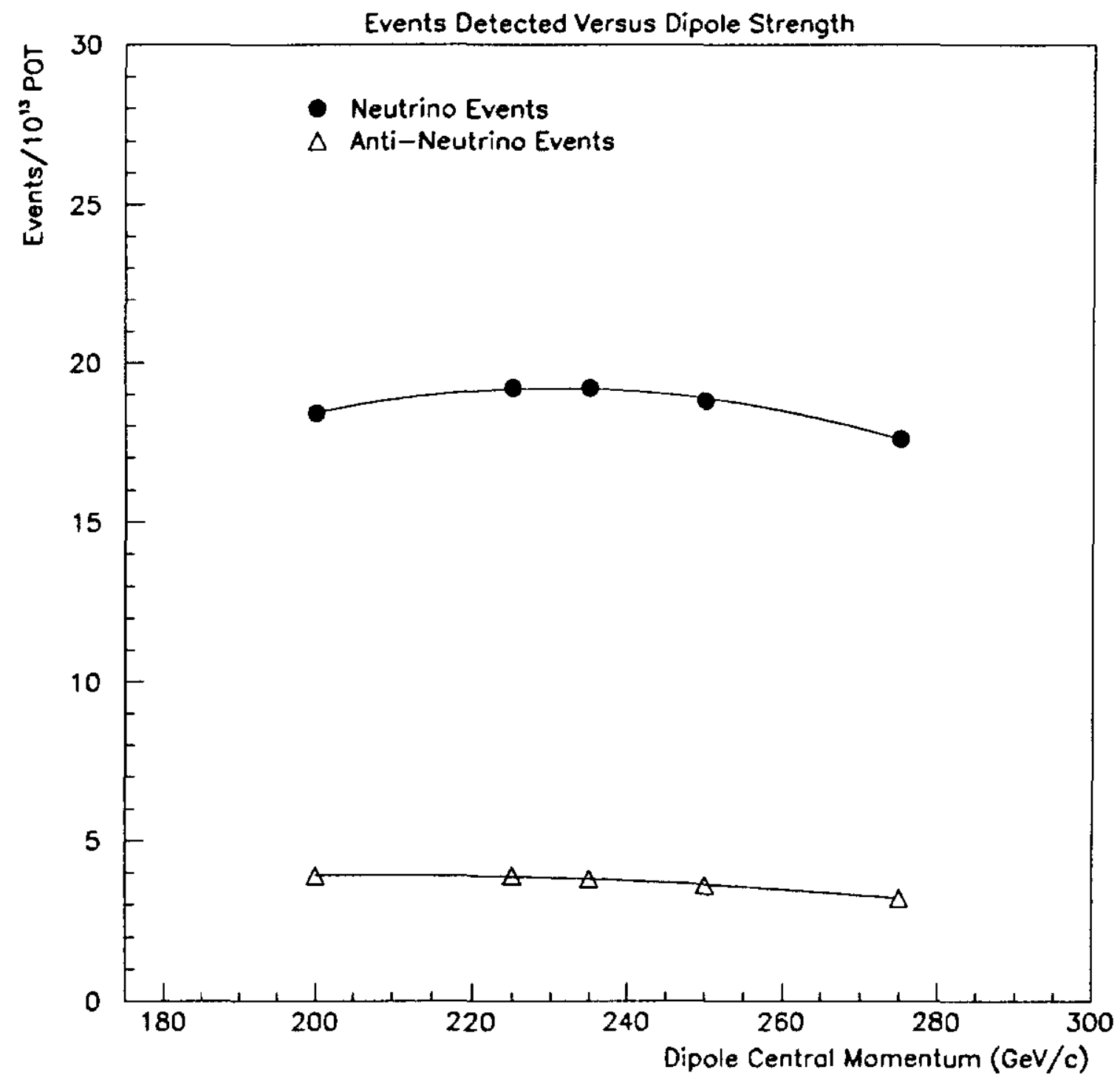

Figure 8: Interaction Rate vs. Field Strength for a $7.8 \mathrm{mrad}$ bend. 


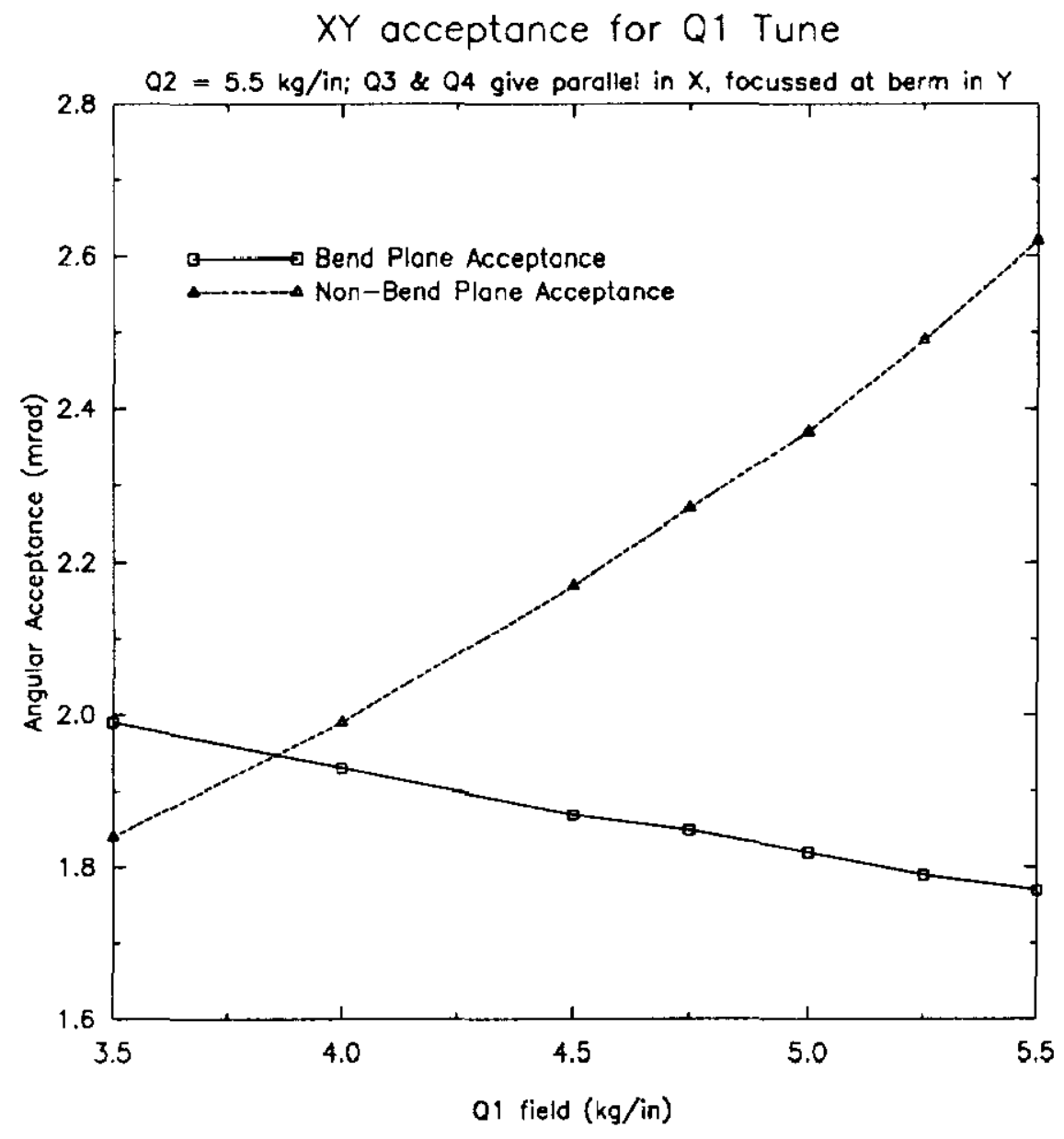

Figure 9: Bend and Non-Bend Plane Angular Acceptance vs. Q1 field. 


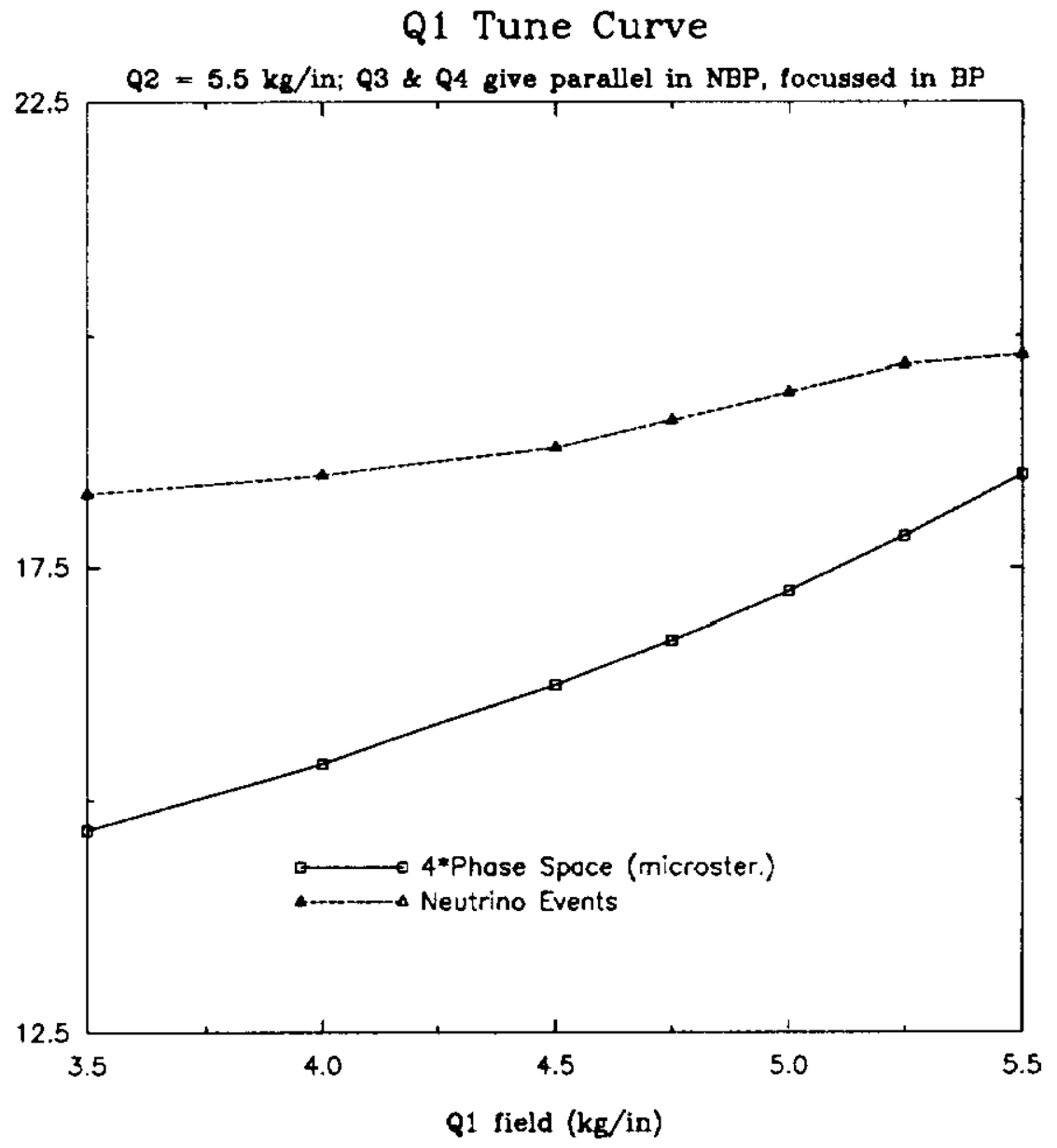

Figure 10: Angular Phase Space and Interaction Rate vs. Q1 Strength. 


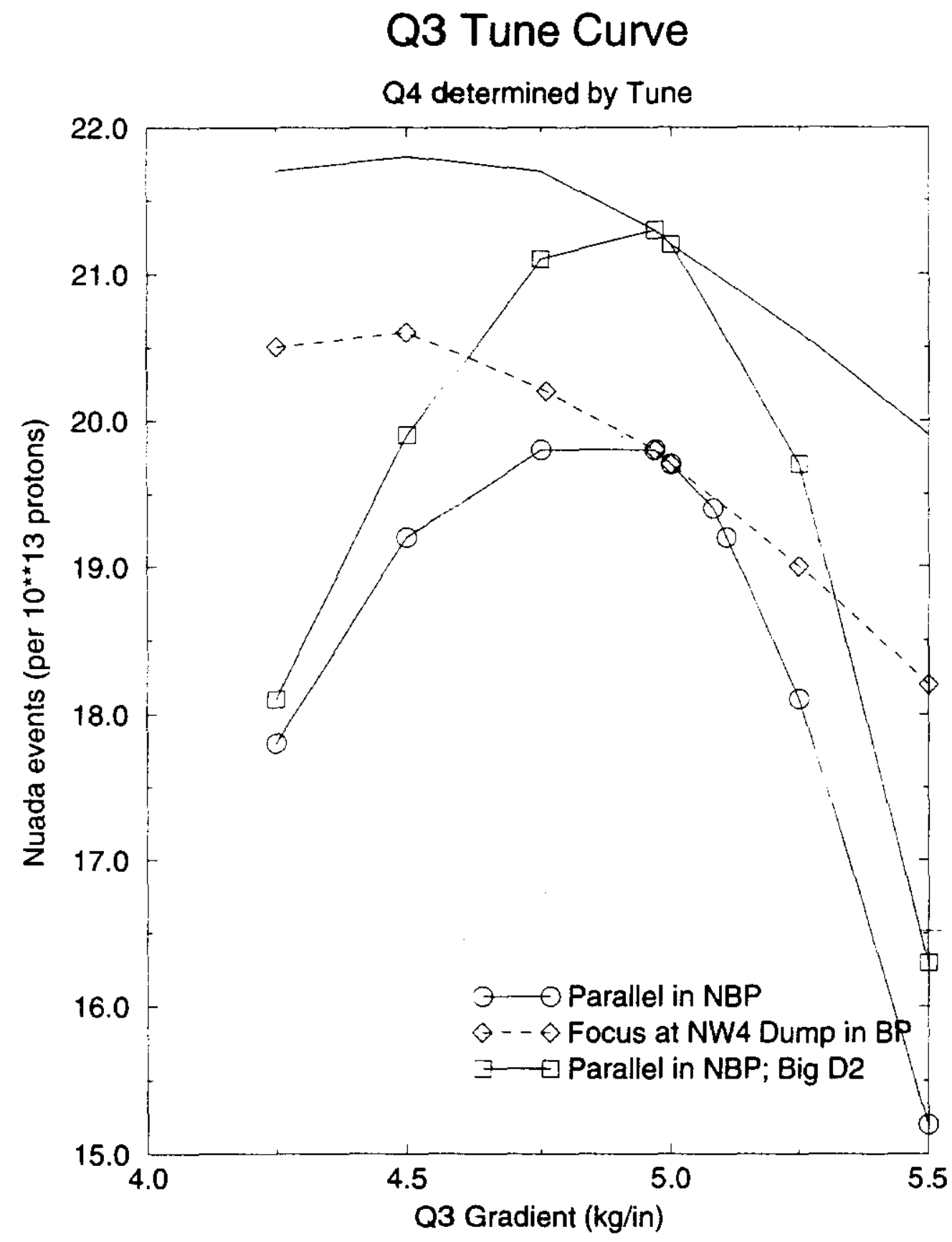

Figure 11: Interaction Rates vs. Q3 Field for Various Beam Tunes. 


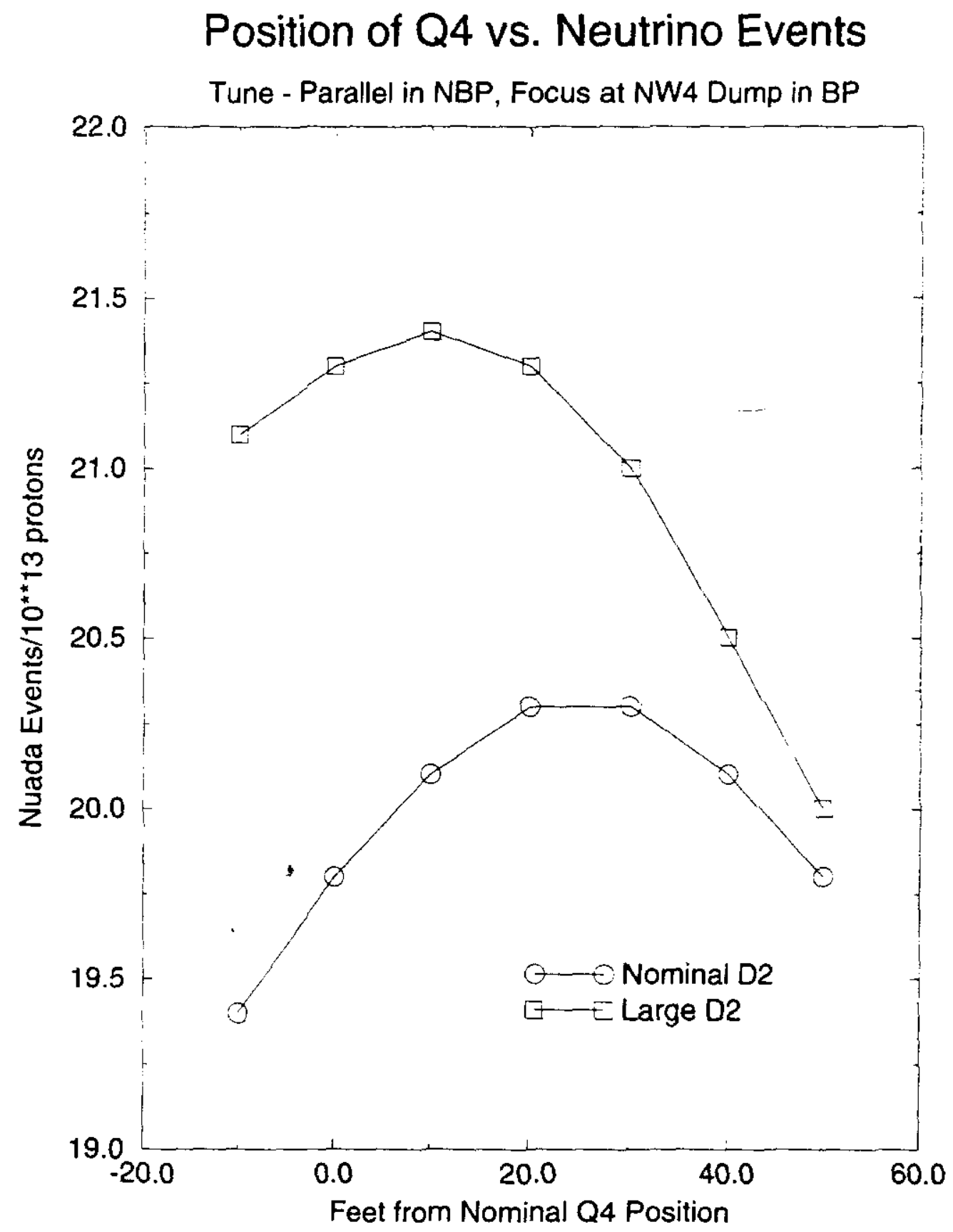

Figure 12: Rate vs. Q4 Position for Standard Tune. 


\section{Position of Q4 vs. Neutrino Events}

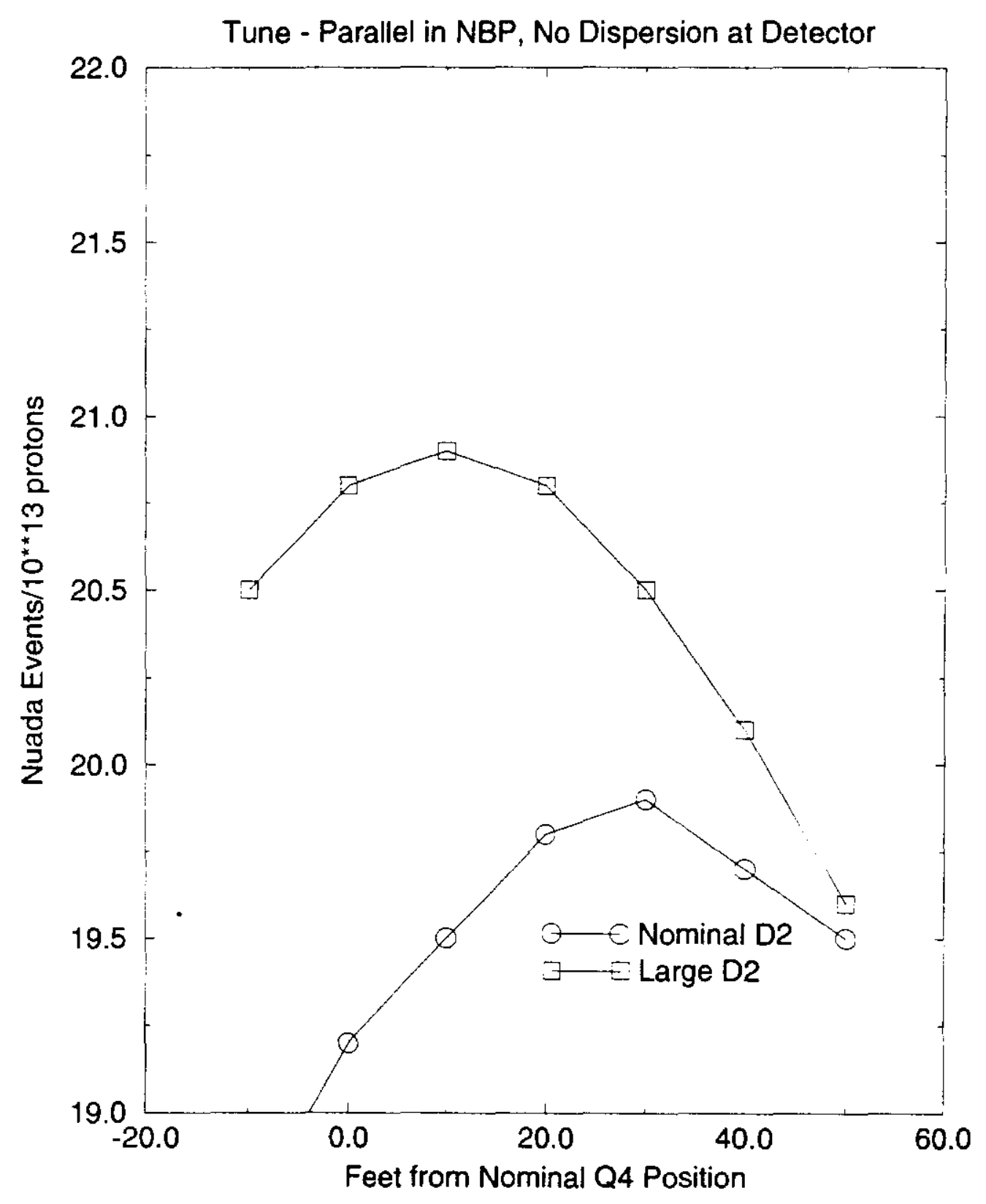

Figure 13: Rates vs. Q4 Position for Achromatic Tune. 

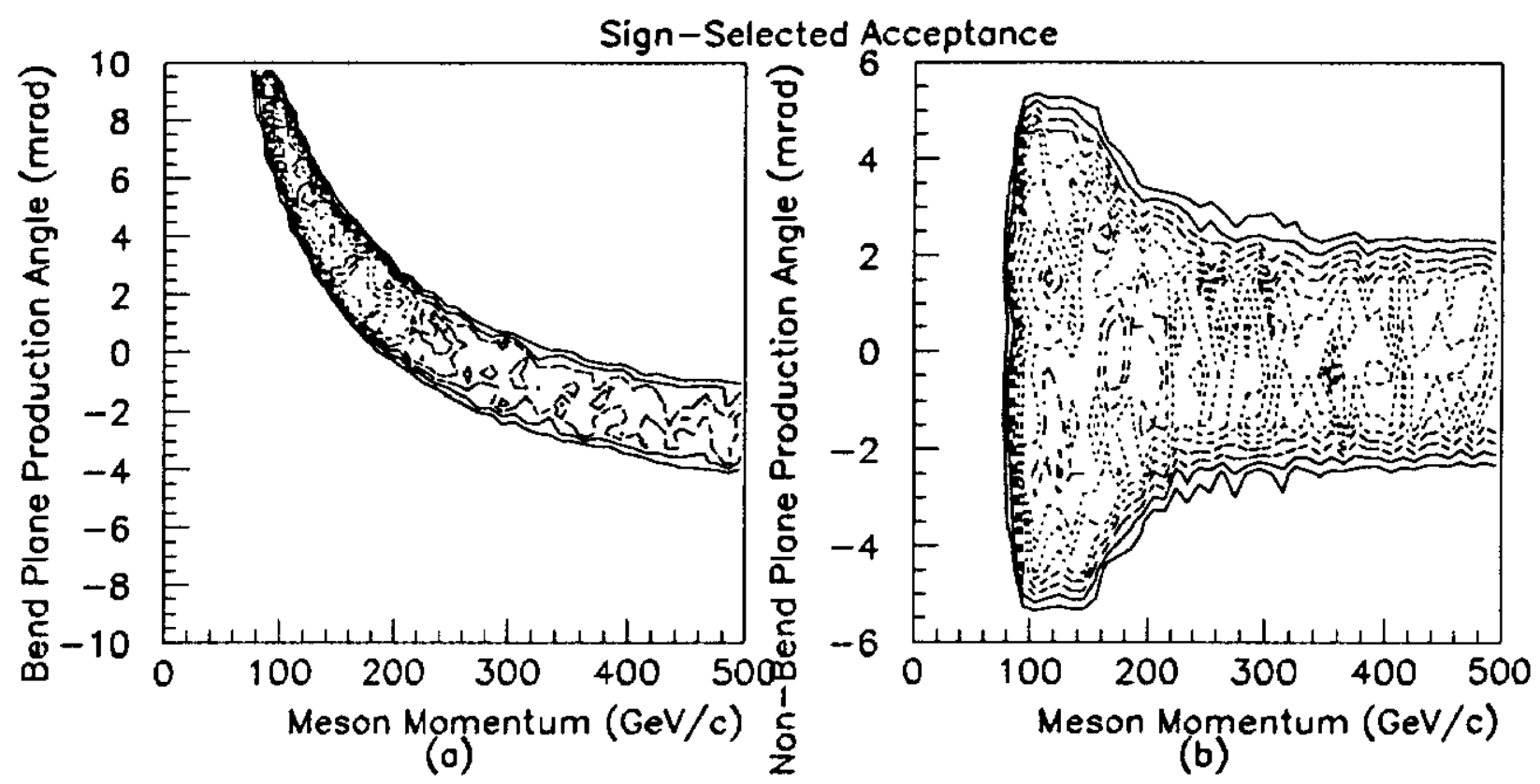

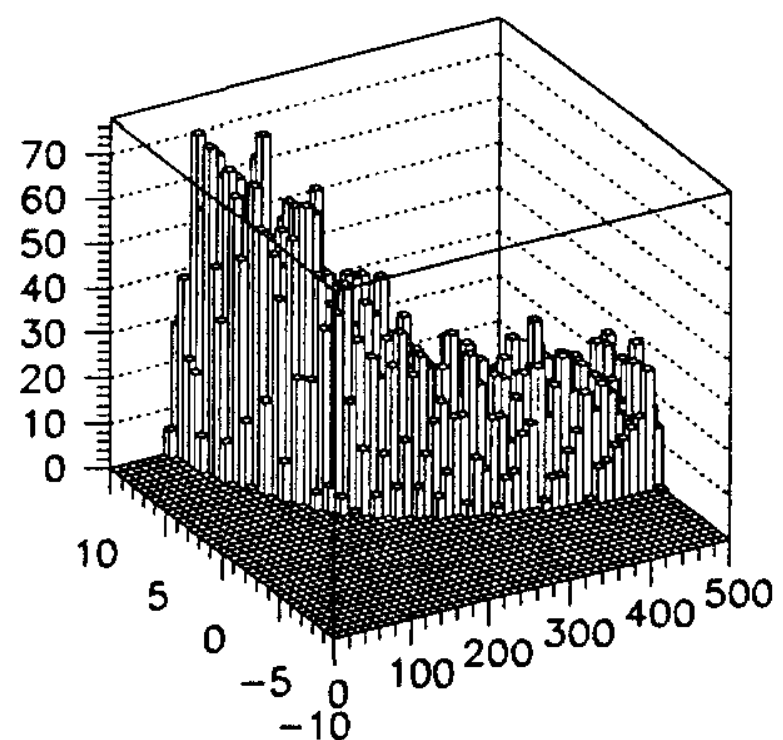

(c)

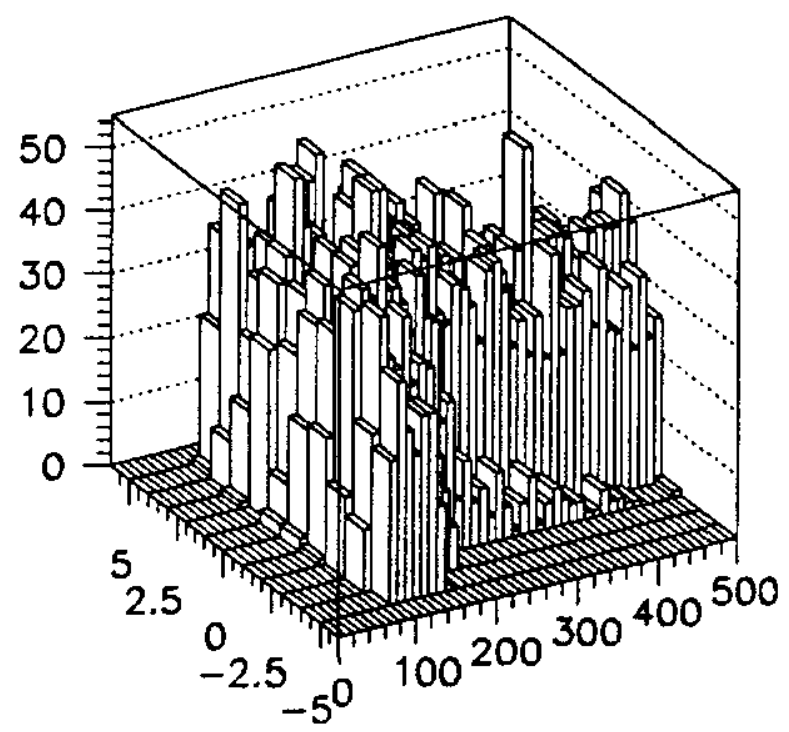

(d)

Figure 14: $p, \theta$ Acceptance of the train for sign-selected mesons in the bend (plots (a) and (c)) and non-bend (plots (b) and (d)) views. The normalization in the lego plots is arbitrary. 


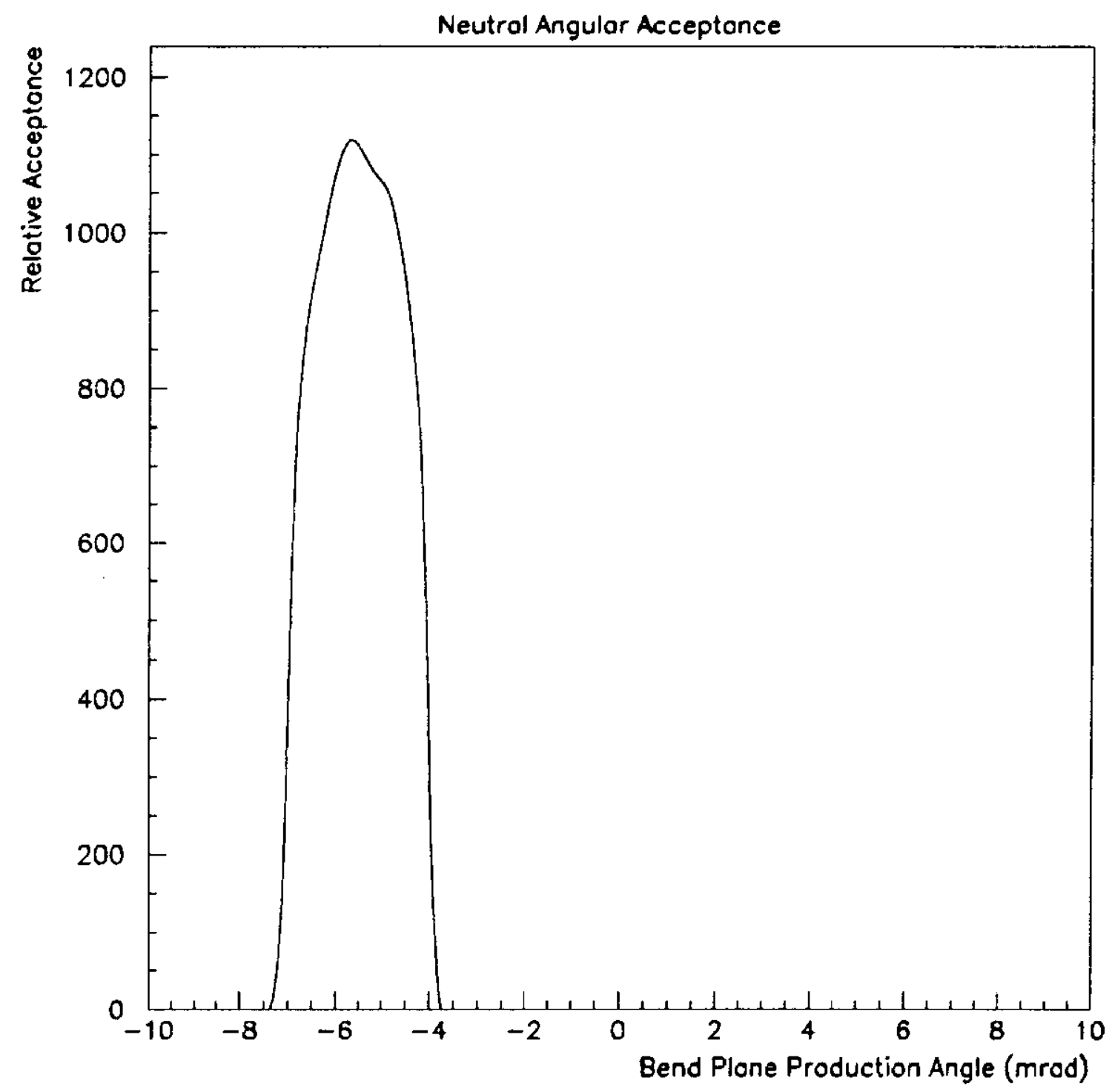

Figure 15: $\theta$ Acceptance of the train for neutrals $\left(K_{L}\right)$-independent of mode. The acceptance at a fixed $\theta$ is independent of momentum for neutrals. 


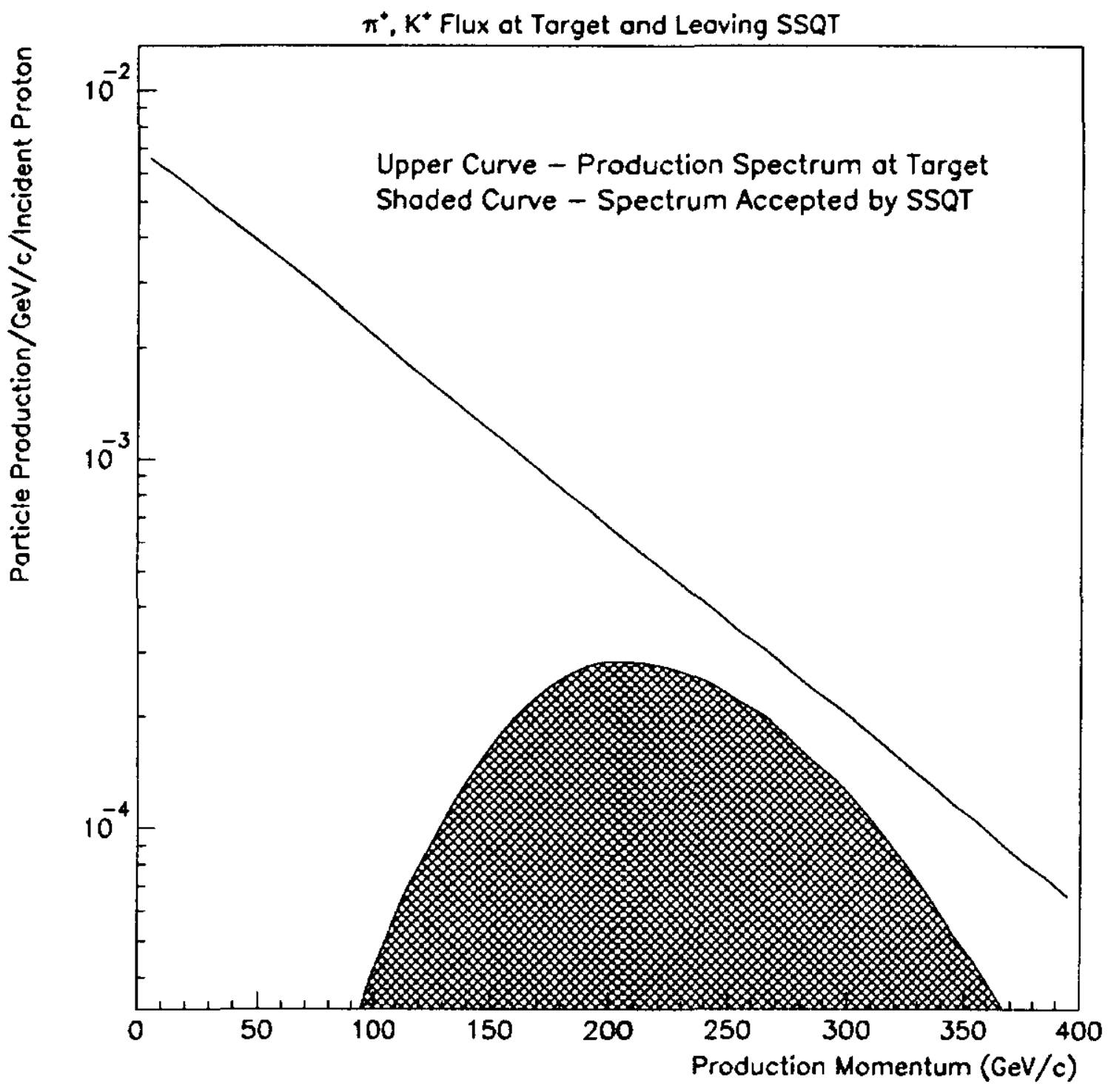

Figure 16: Momentum Spectra of $\pi^{+}, K^{+}$mesons (1) produced at the target and (2) leaving the train, normalized per incident proton (in neutrino mode). The upper curve is the sum of the $\pi^{+}$and $K^{+}$rates, which, when integrated over angle, yields a sum of two exponentials. 


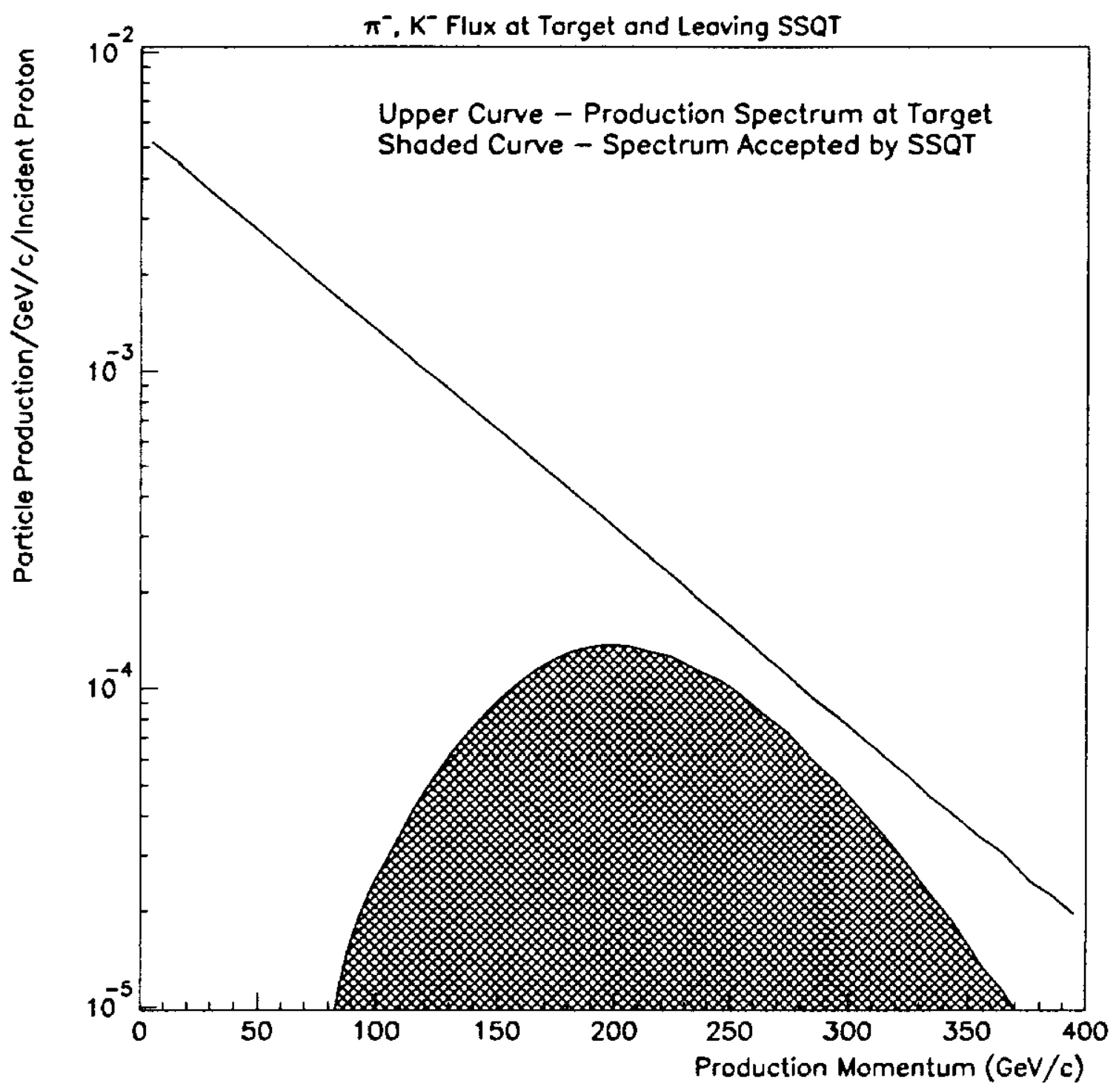

Figure 17: Momentum Spectra of $\pi^{-}, K^{-}$mesons (1) produced at the target and (2) leaving the train, normalized per incident proton (in antineutrino mode). 


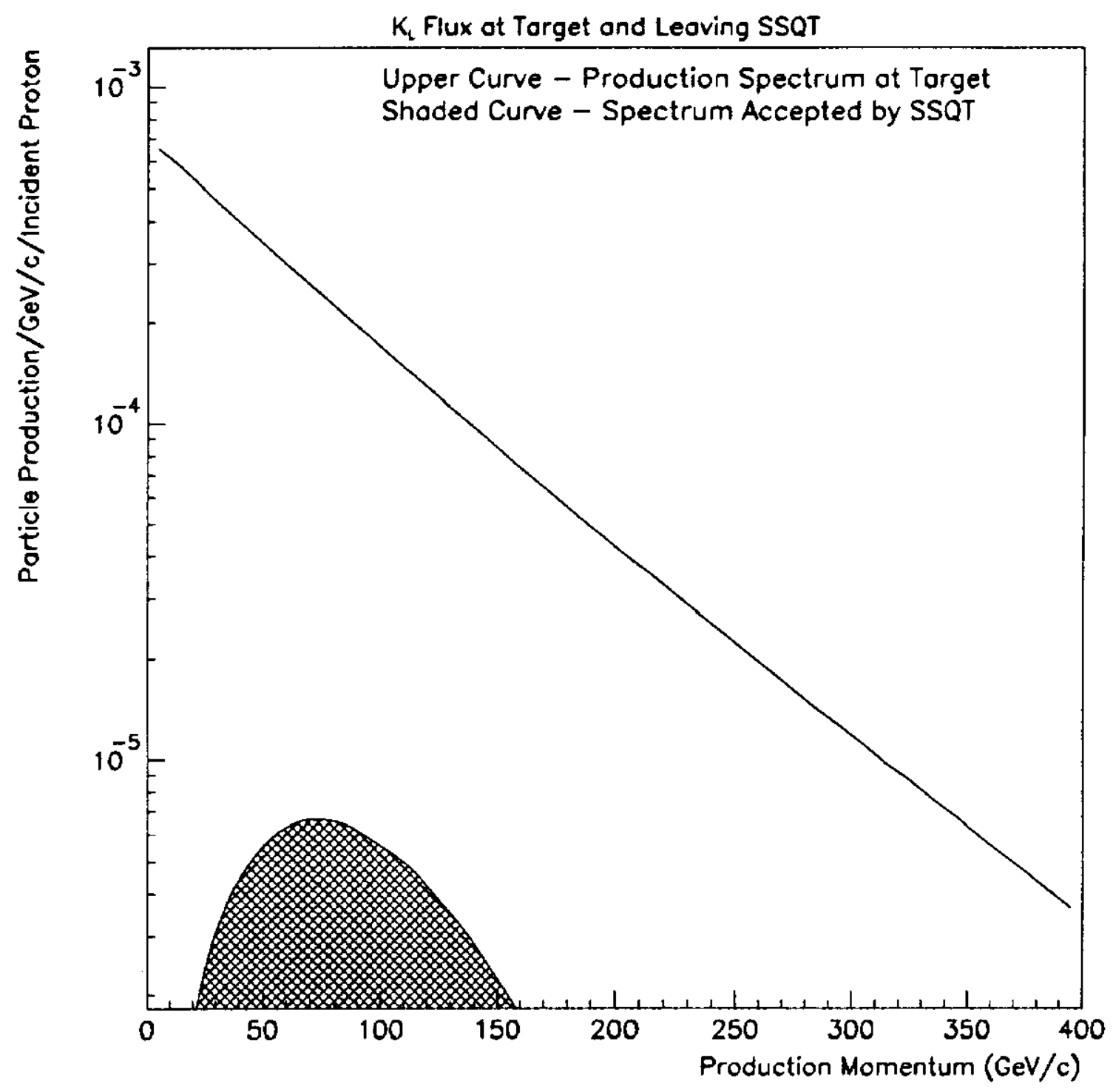

Figure 18: Momentum Spectra of $K_{L}$ mesons (1) produced at the target and (2) leaving the train, normalized per incident proton. 


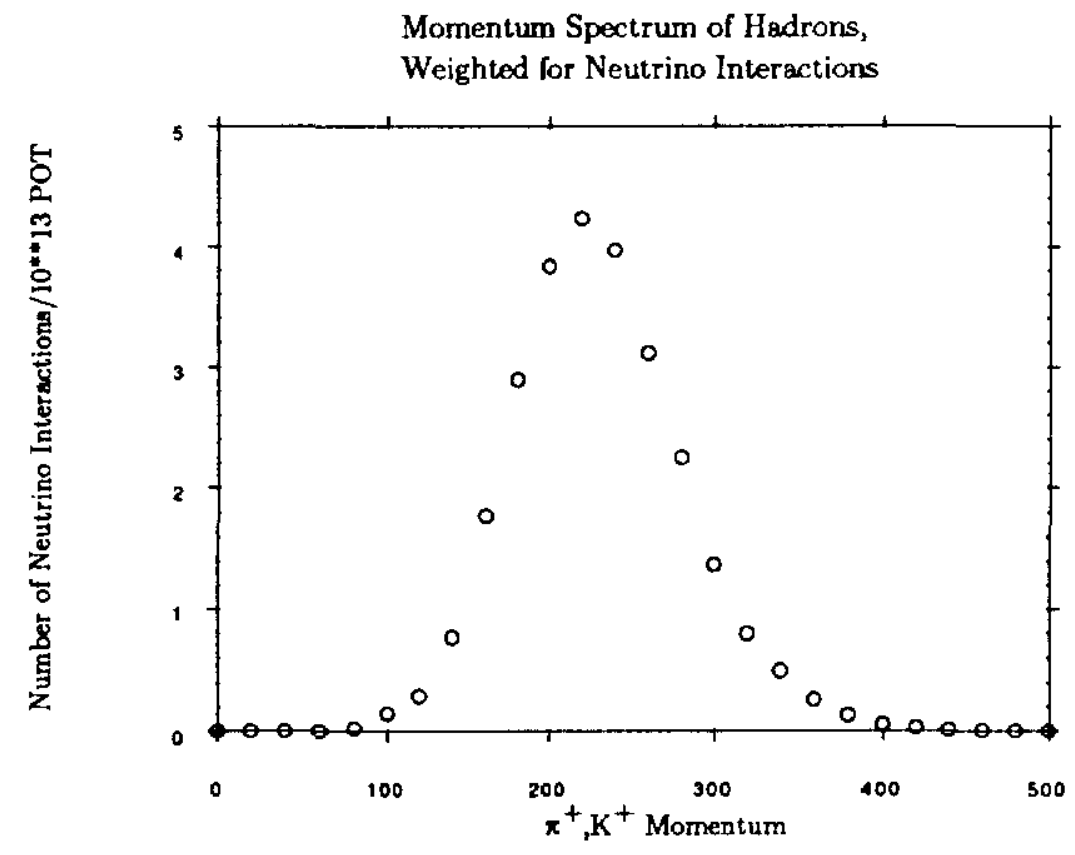

Figure 19: Momentum Spectrum of $\pi^{+}, K^{+}$Mesons, Weighted for Resultant Neutrino Interactions. The corresponding plot for $\bar{\nu}_{\mu}$ is similar in shape but is lower by the ratio of $\bar{\nu}_{\mu} / \nu_{\mu}$ rates. 


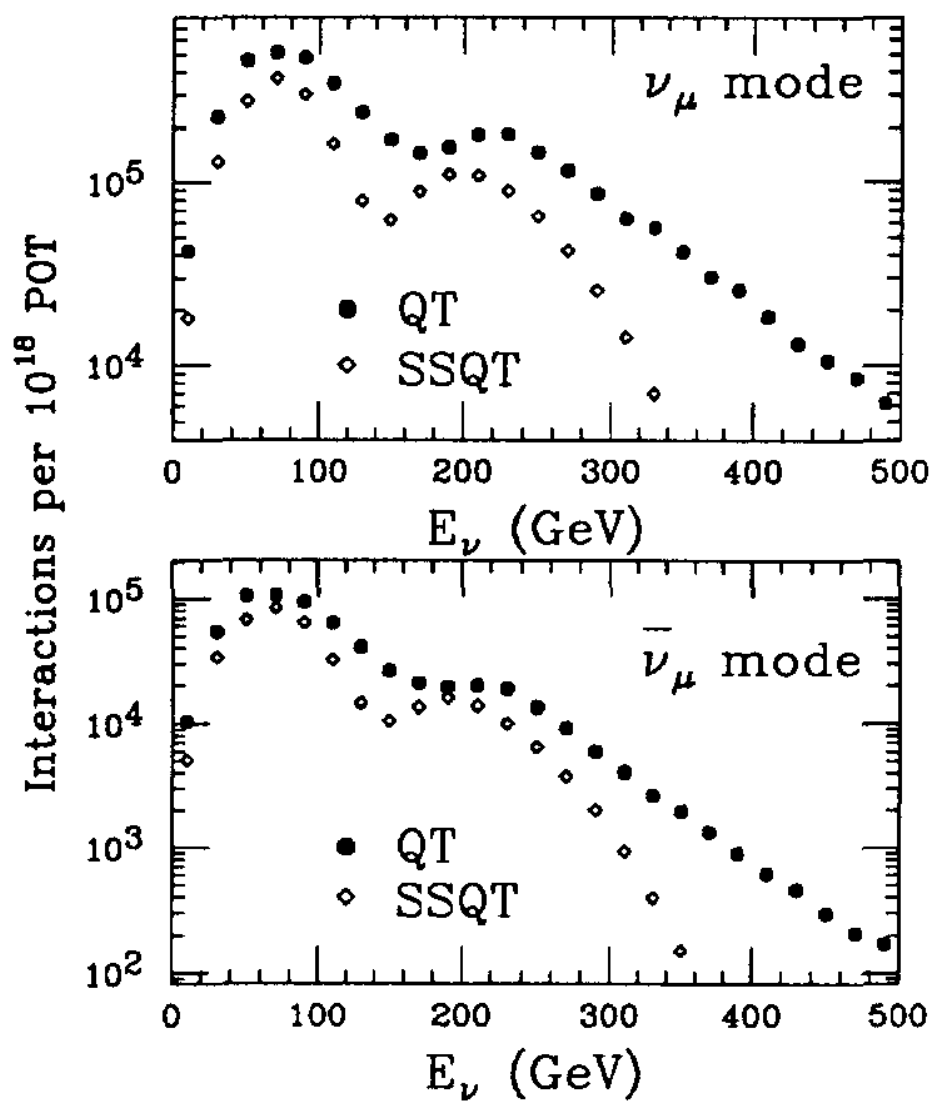

Figure 20: Comparison of the Rate and Spectrum of the SSQT to the E-770 Quadrupole Triplet, for neutrinos and antineutrinos. Events satisfy a $50^{\prime \prime}$ radial cut. 


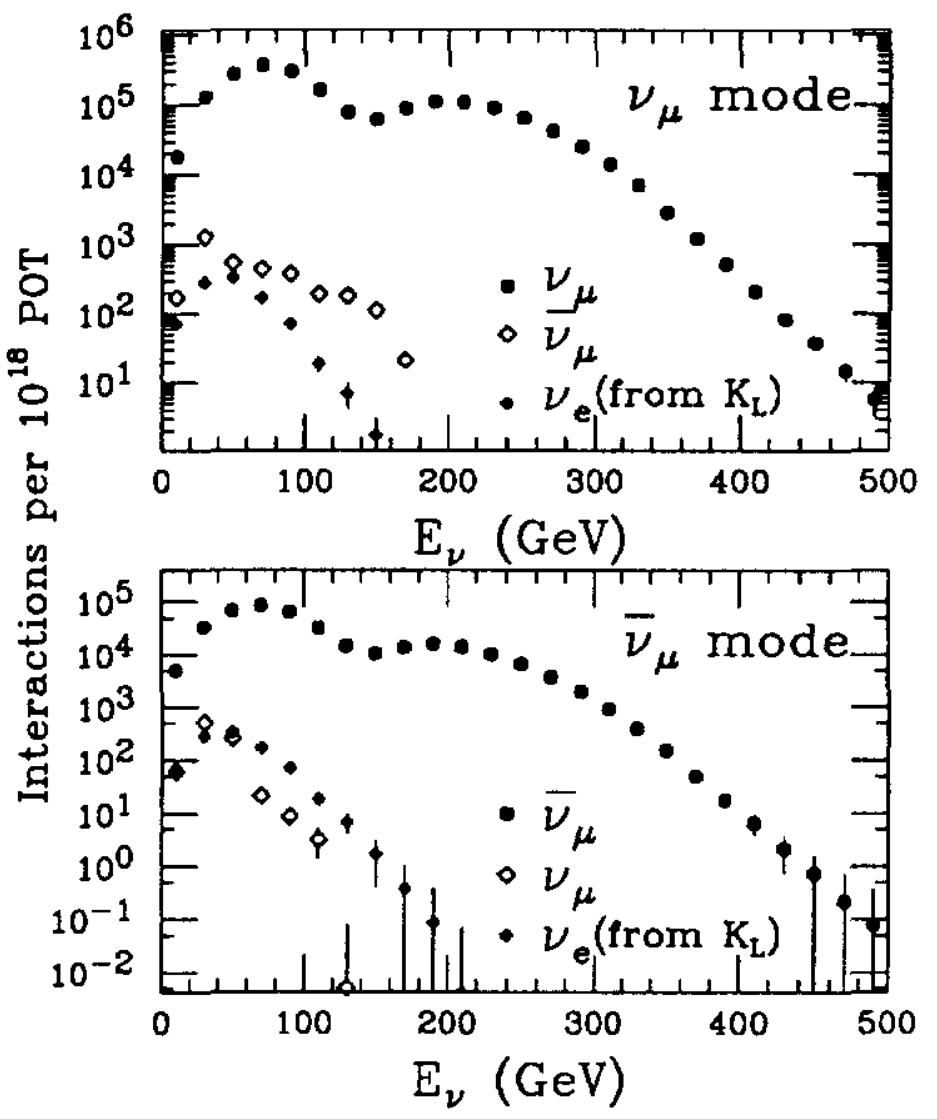

Figure 21: Rates and Backgrounds for $\nu_{\mu}$ and $\bar{\nu}_{\mu}$ modes in the SSQT. All backgrounds have been included. The simulation has a radial cut of $50^{\prime \prime}$. 


\subsection{Dispersion}

Bends produce momentum dispersion in the beam. Our two bends will therefore induce a momentum walk across the face of the detector. The actual distribution of events at any position in the bend plane is a convolution of the production spectrum, the beam optics, and the decay kinematics of the emerging mesons. We can choose either to optimize the rate or to minimize the dispersion in momentum across the face of the detector. The first choice is the best from considerations of both rate and background. We have therefore chosen to center the neutrino distribution on the Lab $\mathrm{E}$ detector by choosing the line from the exit of the final bend to the peak of the impact point distribution at Lab $\mathrm{E}$ to lie along NCenter. We show in Fig. 22 the distribution of events in neutrino mode over the detector in the bend plane. It has a $\sigma$ of approximately 1 meter. Small errors in the final mean position can be removed by a tune of the final bend; the entire detector only has a angular size of about 1 mrad.

The remaining dispersion is acceptable. Fig. 23 shows the mean momentum as a function of position across the face of the detector for the above configuration. It has a walk of about $50 \mathrm{GeV} / \mathrm{c}$ across the detector. Most of this walk occurs where the rate is small, as can be seen by comparing Figs. 23 and 22 .

\subsection{Dumps}

The design of the dumps is a complicated and difficult problem. We must ensure that the resultant protons strike well into the dump. The "rule-of-thumb" is that the impact point must be an inch into the face of the dump, and we have adopted this requirement. ${ }^{7}$ The dumps must also protect personnel and minimize groundwater contamination.

The dumps follow a standard design: $56^{\prime \prime}$ wide by $76^{\prime \prime}$ tall, and $12^{\prime}$ long. The arrangement in the two modes was shown in Fig. 6-7.

The one-inch requirement leads to a small clearance in antineutrino mode. The 250 $\mathrm{GeV} / \mathrm{c}$ central ray is separated from the $800 \mathrm{GeV} / \mathrm{c}$ beam at the first dump by only $1.43^{\prime \prime}$. Given the required one-inch clearance, the $250 \mathrm{GeV} / \mathrm{c}$ beam misses the dump by only $0.43^{\prime \prime}$.

This small distance does not significantly affect the acceptance: the acceptance of the train is reduced by less than $1 \%$ of itself (the occlusion of the dumps has been included in all rate and background calculations). It does place a stringent requirement on the alignment of the dump; an error of $0.1^{\prime \prime}$ inwards would cut more than $10 \%$ of the rate.

The engineering design of the dumps is described in Ref. [9]. We only mention here that

\footnotetext{
${ }^{7}$ The distance from the dump edge to the center line will shrink at $900 \mathrm{GeV}$; we will use GEANT to determine whether the full inch is necessary.
} 


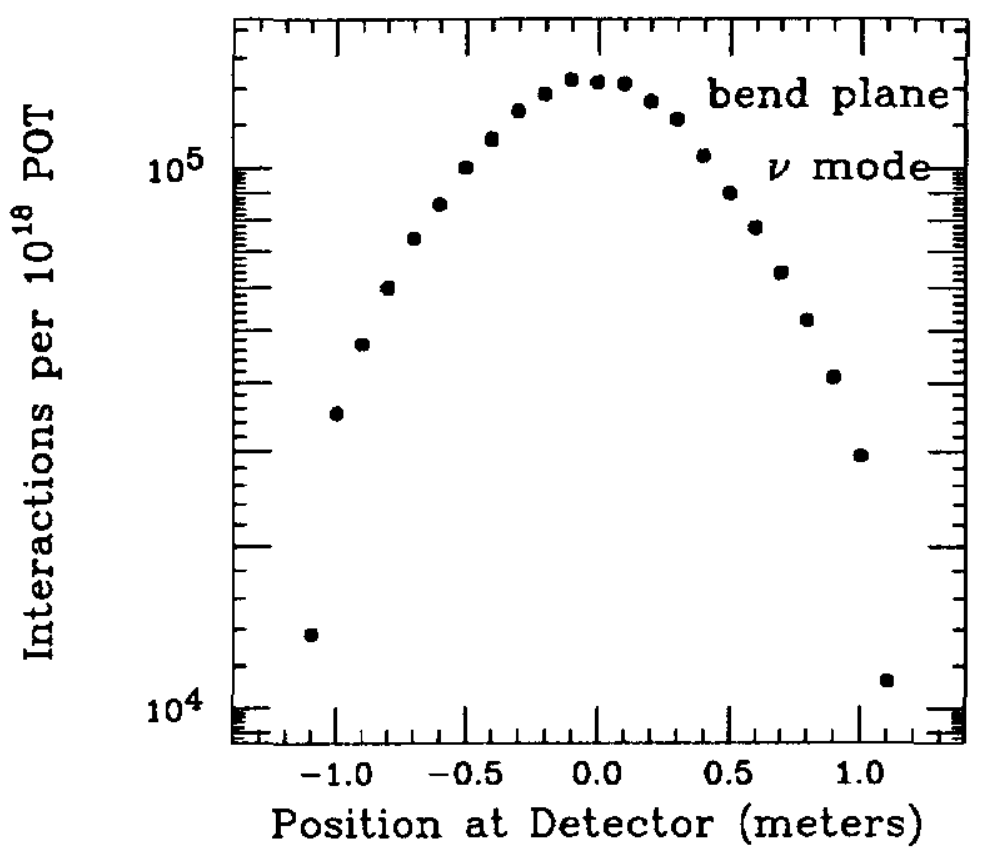

Figure 22: Impact Position of the Neutrinos on the Lab E detector.

the dump can move up and down by up to $2^{\prime \prime}$ so that we can vary the distance from the edge to the dump point and possibly pick up rate in antineutrino mode.

In neutrino mode, the beam hits $3.3^{\prime \prime}$ from the $250 \mathrm{GeV}$ central trajectory. The edge of the dump is then $2.3^{\prime \prime}$ away from the central ray, so the $4 \mathrm{Q} 120$ aperture, and not the dumps, limit the acceptance. 


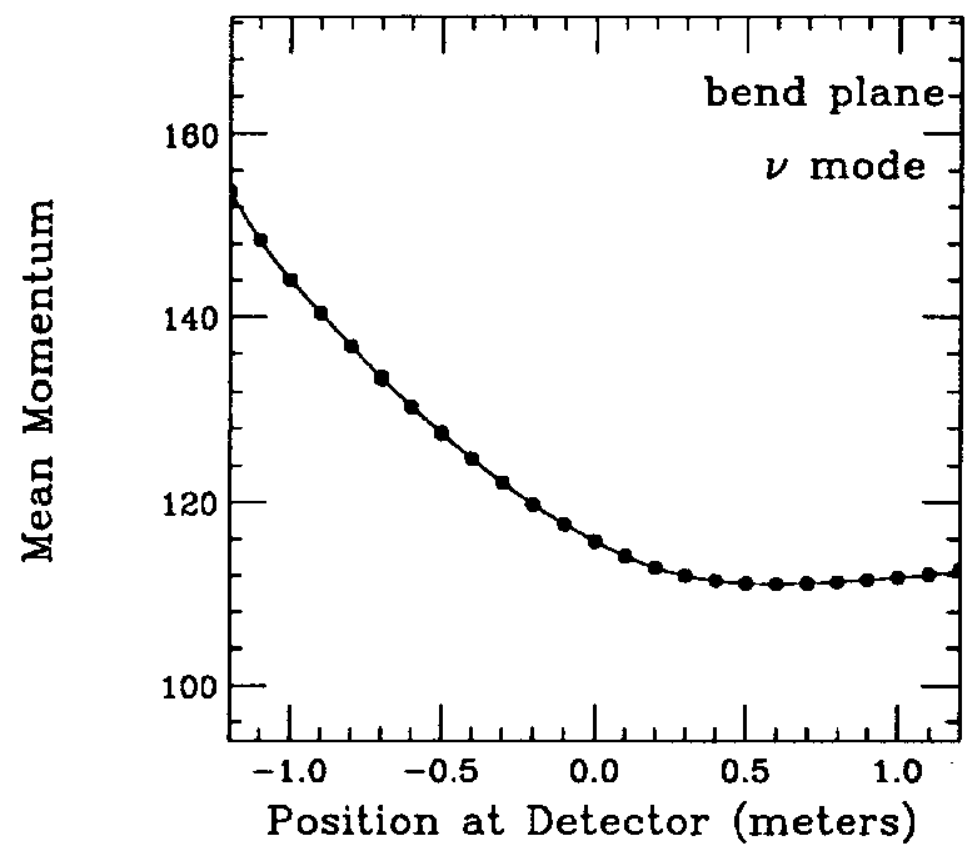

Figure 23: Mean momentum as a function of position in the Lab E detector. Note the suppressed zero.

\section{Backgrounds}

There are two general kinds of beam-related backgrounds:

1. $\nu_{e}$ production

As discussed earlier, $\nu_{e}$ 's directly bias the neutral current signal. $\nu_{e}$ 's come from $K^{ \pm} \rightarrow$ $\pi e \nu_{e}$ and the corresponding semileptonic $K_{L}$ decay. We have excellent control of the first and have lowered the second to an acceptable level.

2. Wrong-Sign Backgrounds

These mix $R_{\nu}$ and $R_{\bar{\nu}}$. However, the wrong-sign contaminations are small and do not significantly affect the measurement. 


\subsection{Beam Simulation Codes}

Four programs have been used to study the rates and backgrounds: NUADA, TURTLE, GEANT, and the E-770 beam simulation program described in Sec. 3.2. The rates and spectra of the $\nu_{\mu}$ and $\bar{\nu}_{\mu}$ signals agree to within a few percent.

NUADA and TURTLE are the standard codes used at FNAL for beamline development. They have been adapted to the SSQT with second-order optics. Both use differential crosssections from Malensek[7] for particle production.

The E-770 code used the data to extract differential cross-sections instead of taking them from an external source. The data came from the decays $\pi, K \rightarrow \mu \nu_{\mu}$; the $\nu_{\mu}$ are the charged-current signal. A first-order simulation of the beamline was fed into the E-770 physics Monte Carlo where each neutrino had a weight proportional to the differential crosssection of its parent meson (normalized to the data, so no absolute flux information was required). The parameters included in the calculation of the weight were the momentum and production angle of the parent meson, and the impact point of the primary proton on the target. The program then fit $d^{2} N / d p d \Omega$ by varying the coefficients of the parameterization and the shape of the primary beam. The agreement is shown in Fig. $24 .{ }^{8}$ This Monte Carlo was then modified for the SSQT to predict the rates and backgrounds expected for E-815. ${ }^{9}$

A GEANT simulation has also been written for the beam. Only GEANT is able to calculate rescatters and is therefore used for our scraping calculations. Since GEANT's power makes it slow, we have not been able to match the precision of the other programs. As a check, various generators (FLUKA, GHEISHA, and HERWIG) have been tried so that we can study the variation of the rates with generator. Some variations of the background rate span a factor of three; the results presented in this memorandum come from the FLUKA generator and are the highest of any version, which still induces a negligible error.

\section{2 $\nu_{e}$ Production in the Beam}

\subsection{1 $\quad K^{ \pm} \rightarrow \pi e \nu_{e}$}

The E-770 measurement of $\sin ^{2} \theta_{W}$ had the $K^{ \pm} \rightarrow \pi e \nu_{e}$ background discussed in Sec. 1.1: charged-current $\nu_{e}$ were indistinguishable from neutral current $\nu_{\mu}$ because the showering electron was lost in the shower of the fragmented nucleon. We predicted the $\nu_{e}$ rate from this source from the $K^{ \pm} \rightarrow \mu \nu_{\mu}$ data using the E-770 code described in Sec. 3.1: the fitted

\footnotetext{
${ }^{8}$ The disagreement with the Atherton data is only $10-15 \%$.

${ }^{9}$ In this case, the production spectra come from Refs. [7] and [5].
} 


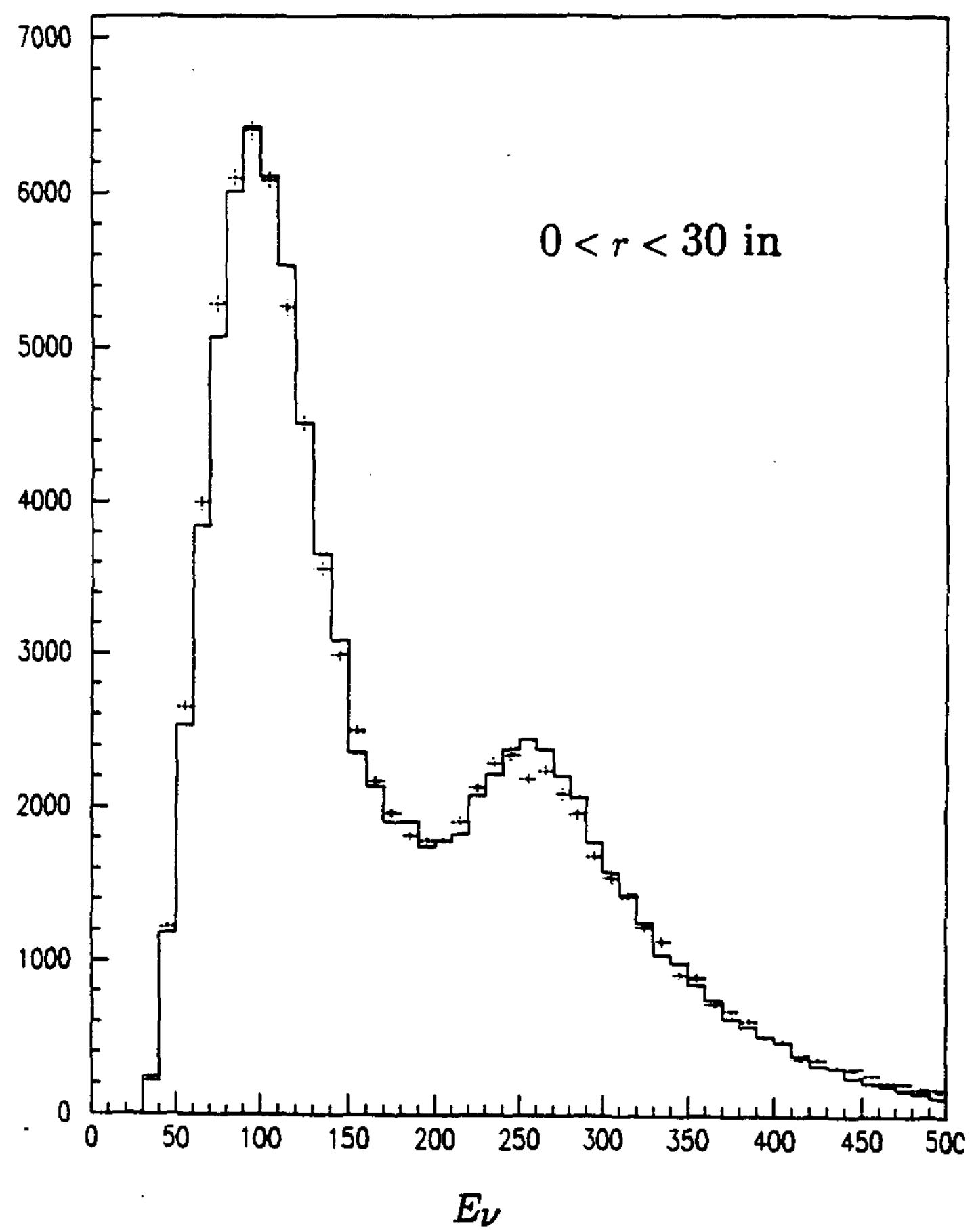

Figure 24: Comparison of Beam Simulation program to E-770 Data. The histogram is the data, and the crosses are the simulation. 


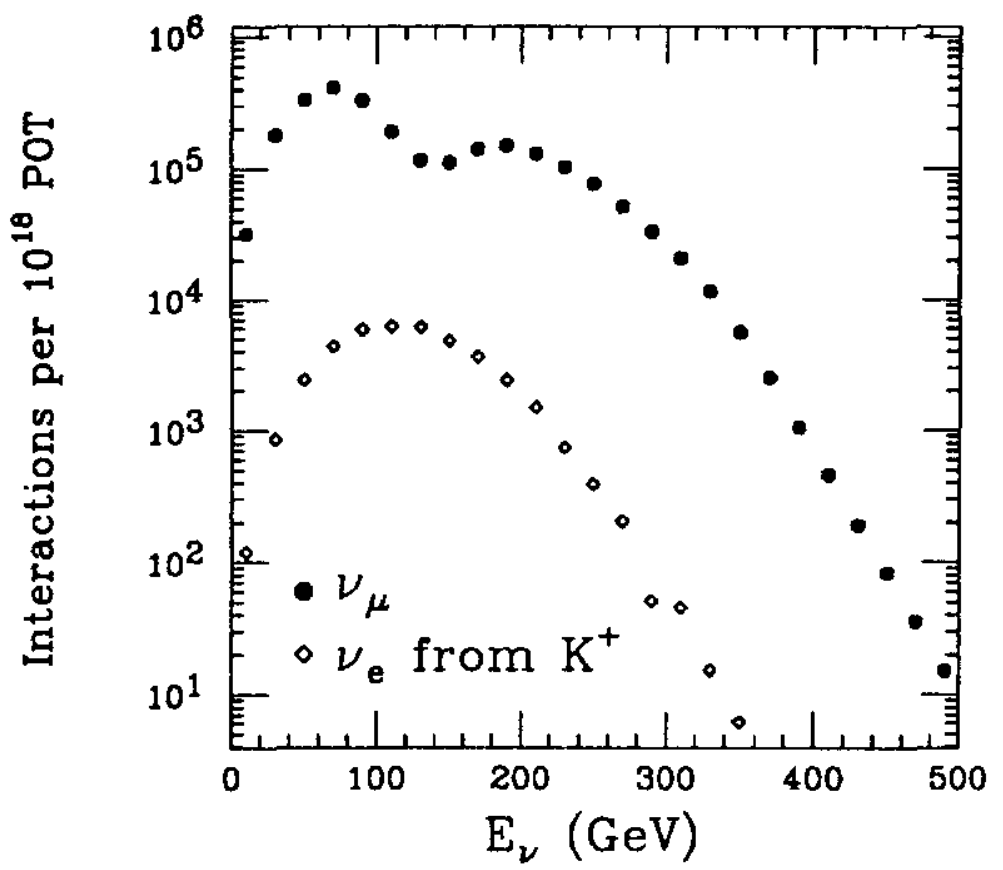

Figure 25: Relative Spectra of (1) $\nu_{\mu}$ from all charged current decays, and (2) $\nu_{e}$ from $K \rightarrow \pi e \nu_{e}$ for neutrino mode.

$d^{2} N / d p d \Omega$ for $K^{ \pm}$was used to predict the rate $K^{ \pm} \rightarrow \pi e \nu_{e} \cdot{ }^{10}$ The agreement in the spectra was shown in Fig. 24. A $\nu_{e}$ to $\nu_{\mu}$ comparison for this source is given in Fig. 25.

This technique is not guaranteed. If the detected $\nu_{e}$ 's from $K e 3$ decays come from a poorly determined portion of the $K^{ \pm}$spectrum, then this procedure leads to a large uncertainty in the $\nu_{e}$ flux. Fig. 26 shows the $K^{+}$-momentum for those $K$ 's which produce $\nu_{\mu}$ accepted in the detector, together with those which produce accepted $\nu_{e}$. The substantial overlap demonstrates the reason that fitting $K^{ \pm} \rightarrow \mu \nu_{\mu}$ is a good prediction of $K^{ \pm} \rightarrow \pi e \nu_{e}$. Quantitatively, we found that varying the parameters of the E-770 fit within the allowed errors yielded an uncertainty of $\delta\left(\sin ^{2} \theta_{W}\right)< \pm 0.0005$. Most of that error came from lack of knowledge of the beam spot size and exact locations of the magnets, problems that will be greatly reduced in $\mathrm{E}-815$. The $\nu_{e}$ final error in $\mathrm{E}-815$ will be dominated by the $K^{ \pm} \rightarrow \pi e \nu_{e}$

\footnotetext{
${ }^{10} \nu_{e}$ from $\pi \rightarrow e \nu_{e}$ decays are also included through the simulation. $\nu_{e}$ from charm, $\Lambda$ 's, and other sources have been calculated and are negligible.
} 


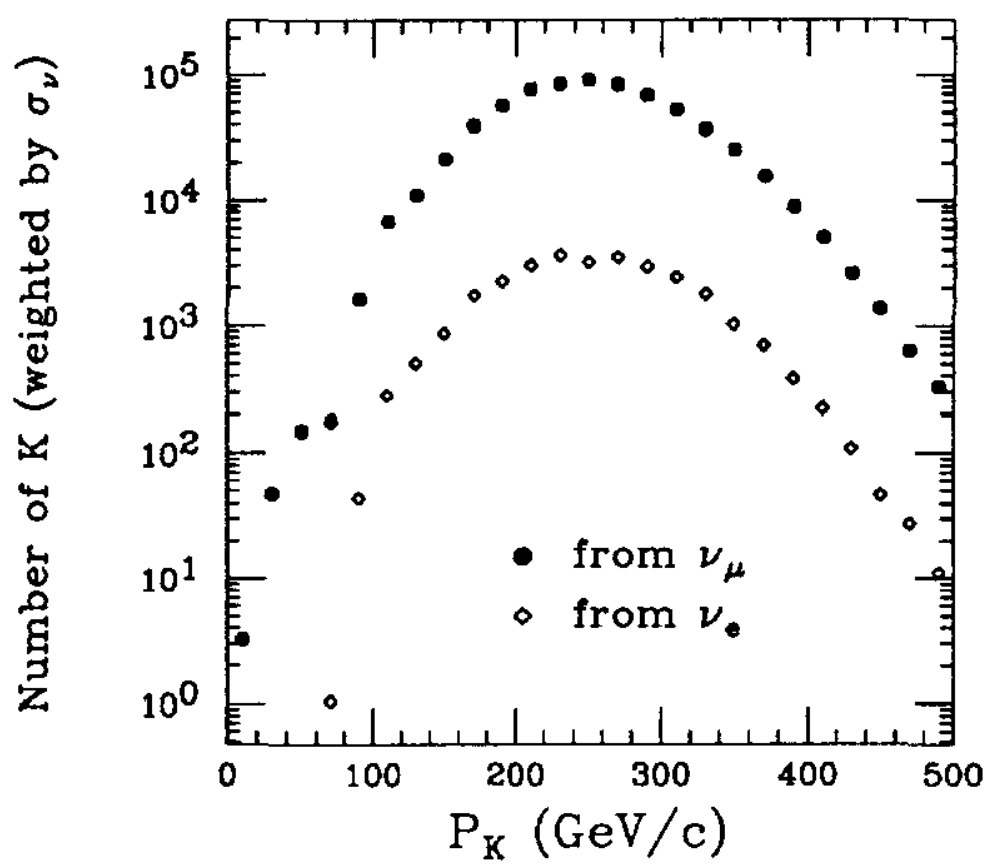

Figure 26: Overlap in $K^{ \pm}$momentum for $K^{ \pm} \rightarrow \mu \nu_{\mu}$ and $K^{ \pm} \rightarrow \pi e \nu_{e}$. The curve labeled $\nu_{\mu}$ is the momentum spectrum of $K^{+}$responsible for accepted $\nu_{\mu}$; the curve labeled $\nu_{e}$ is the momentum spectrum of $K^{+}$responsible for $\nu_{e}$.

branching ratio which translates into an error $\delta\left(\sin ^{2} \theta_{W}\right)= \pm 0.0007 .{ }^{11}$

\subsection{2 $\quad K_{L} \rightarrow \pi e \nu_{e}$}

The second source of $\nu_{e}$ production is $K_{L} \rightarrow \pi e \nu_{e}$. In theory we could measure this from the corresponding $K_{L} \rightarrow \pi \mu \nu_{\mu}$ decay but the latter constitutes only $0.8 \%$ of the charged current data and is too small to be measured reliably. We have used a parameterization based on data from Skubic et al.[5] which measured $K_{S}$ production from $400 \mathrm{GeV}$ protons on a Be target. The $x_{F}$ and $p_{T}$ range of that data match our accepted $K_{L}$ well $\left(0.15<x_{F}<0.75\right.$ and $p_{T}<2 \mathrm{GeV}$ ). The error in the absolute level is at least $7 \%$ from solid angle, and while the other errors are hard to estimate, we believe that an error of $\pm 20 \%$ on the overall level is conservative. The other source of data is from the CP-violation experiments at FNAL. They are limited to $K_{L}$ produced at $\approx 5 \mathrm{mrad}$ and have not been concerned with a precision measurement of the absolute cross-section. The Skubic parameterization, applied to their

\footnotetext{
${ }^{11}$ To first-order, we need not even exactly reproduce the spectrum: only the total number of $\nu_{e}$ within our cuts is necessary in calculating the NC contamination.
} 
beam and apertures, is $15 \%$ higher than the best estimate from $\mathrm{E}-731 .[8]$

\subsection{Production of $K_{L}$ in Dumps, Windows, Flanges, and Pipes}

Secondary targets exist all along the beamline. Showering can produce either wrong-sign backgrounds or serve as sources of $\nu_{e}$. We have written a GEANT simulation of the beamline to calculate these sources. The other Monte Carlos do not include showering but have been used to check GEANT's prediction for the number and spectra of mesons and protons hitting pipes, windows, and flanges.

The scraping interactions have been estimated by GEANT from each of the obstructions. We find no significant backgrounds from any source.

\subsubsection{Production in Dumps}

After the non-interacting primary protons hit the dump, they will shower and produce $K_{L}$. Some of these may exit the dump and decay which can produce $\nu_{e}$ heading toward the detector. The contribution will be reduced if the $K_{L}$ can re-interact within the dump before escaping. This is where the one-inch rule becomes important.

GEANT predicts that fewer than $3 \times 10^{-4} \nu_{e}$ from $K_{L}$ per $10^{13}$ POT in neutrino mode, about a factor of thirty lower than the "pure" $K_{L}$ produced at the target; in antineutrino mode, the rate is lower because the dump is farther upstream: $2 \times 10^{-5} \nu_{e}$ per $10^{13}$ POT.

\subsubsection{Production in Windows}

All windows along the $\mathrm{NC}$ line decay volume total much less than $1.0 \%$ of an interaction length. The window at the target tube/decay pipe interface has been changed from earlier designs, which presented a 4 " window with a thick $\mathrm{Al}$ flange surrounding it.

A GEANT calculation tells us that the contribution from all windows to either the $\nu_{e}$ rates is less than $1 \%$ of the $K_{L}$ from the target, and is hence negligible.

\subsubsection{Production in Flanges and Pipes}

We have determined the $\nu_{e}$ production from $K_{L}$ created at the flange surrounding the $17^{\prime \prime}$ window (at the Decay Pipe/Target Tube transition) using both the standard beam simulation and GEANT. We note that the flange thickness is under $1 "$, or $7 \%$ of an interaction length. 
We have simulated the production of $\pi$ and $K$ mesons and of secondary protons at the target and propagated them to the flange. With these assumptions, there are $3.1 \times 10^{-5} \nu_{e}$ from $K_{L}$ hitting Lab E with a mean energy of $40 \mathrm{GeV}$. When compared to the $0.9 \times 10^{-2} \nu_{e}$ events from $K_{L}$ produced at the target, this yields

$$
\frac{\nu_{e}\left(K_{L}, \text { flange }\right)}{\nu_{e}\left(K_{L}, \text { target }\right)}=0.3 \%
$$

which is small compared to the $20 \%$ error on the $K_{L}$ fraction.

The largest source of scraping background comes from interactions in the walls of the Decay Pipe. This source increases the $\nu_{e}$ interaction rate from $K_{L}$ decays by $20 \%$ of itself. This small addition to an already small background can be calculated to the required accuracy because the total correction is equivalent to a shift of only 0.0003 in $\sin ^{2} \theta_{W}$.

\subsection{Wrong-Sign Backgrounds}

Wrong-sign backgrounds from the target are small because of the two bends. The wrongsign rate is dominated by scraping of secondaries which hit the downstream vacuum pipe. Fig. 27 shows the position of scraping interactions. The $p, \pi^{ \pm}$, and $K^{ \pm}$'s which hit the pipe are pointed at a relatively shallow angle $(\approx 1 \mathrm{mrad})$ and shower along the length of the pipe.

Quantitatively, we find:

1. Some wrong-sign mesons are produced at the target; these are the remaining ( $\pi^{-}$and $K^{-}$mesons which manage to escape into the train and then decay before the second bend. These yield $0.2 \times 10^{-2} \bar{\nu}_{\mu}$ at a mean energy of $44 \mathrm{GeV}$, compared to the 20.0 expected $\nu_{\mu} / 10^{13}$ POT.

2. Wrong-Sign mesons from scraping are approximately five times as large: $0.01 \bar{\nu}_{\mu} / 10^{13}$ POT.

The energy spectrum of the wrong-sign background interactions in the detector was given in Fig. 21. Wrong-sign production at this level is not a significant source of error for the $\sin ^{2} \theta_{W}$ or $\rho$ determination.

\subsection{Calibration Runs}

It would be desirable to perform in situ checks of the background levels determined through simulations. As a check of the GEANT calculations, we can run with the target out and check the production in the dump. We expect to run long enough to determine the level is acceptably small, which should only take a few days of running time. 

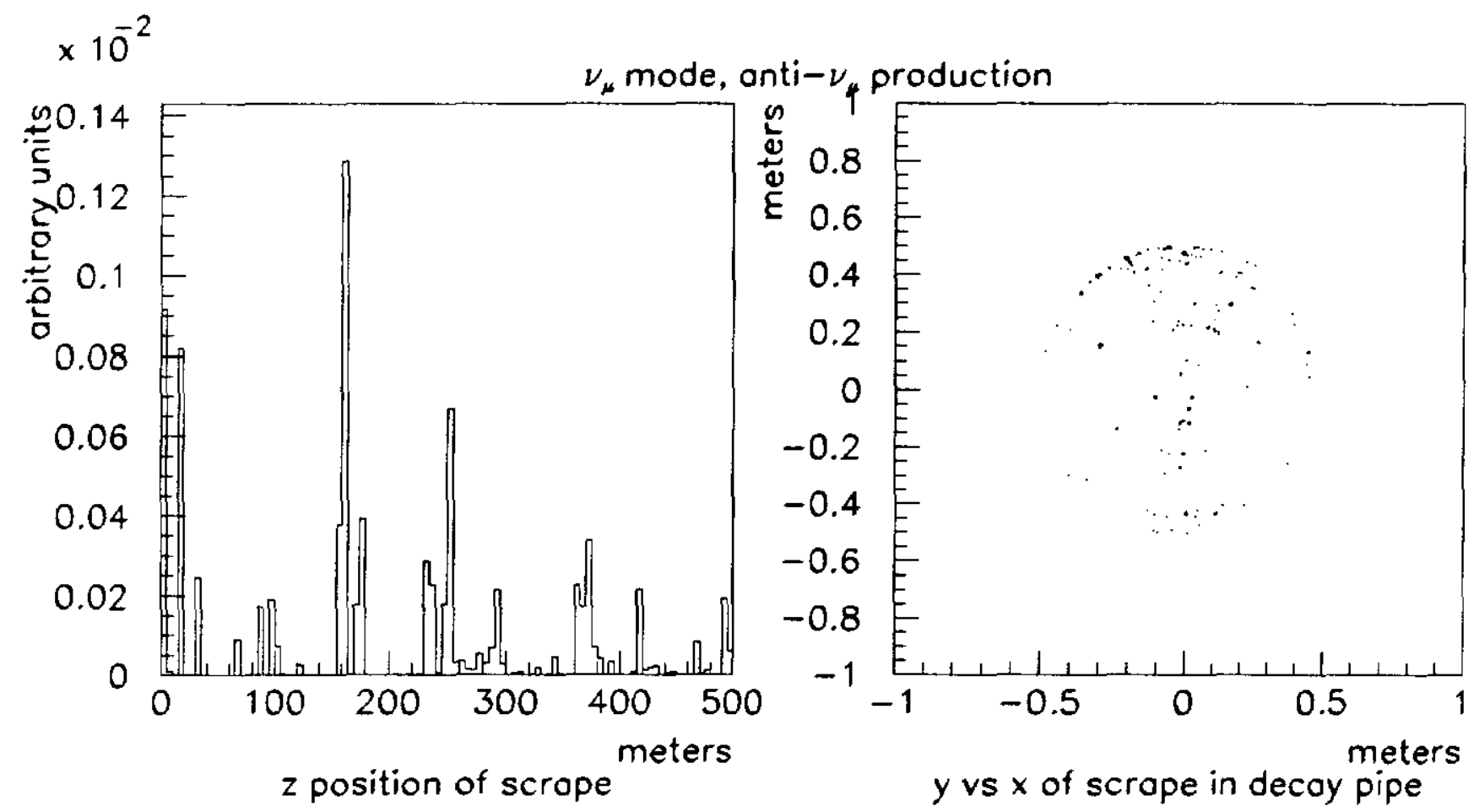

Figure 27: Position of Scraping Interactions. 


\section{Beam Monitoring and Alignment}

\subsection{Primary Beam Monitoring}

Beam Monitoring will be used to assist in the simulation of the $K^{+} \rightarrow \pi^{\circ} e \nu_{e}$ background. The size and shape of the primary proton beam spot affects the $K^{ \pm}$spectrum as shown in Fig. 28. We have varied the spot from a $\delta$-function to a Gaussian with $\sigma_{x}=0.7, \sigma_{y}=3 \mathrm{~mm}$ with the same center. The upstream monitoring scheme will measure the spot shape and location during the run and we will use it as a direct input to the analysis.

It should be stressed that the old E-770 analysis had no input from bearn monitoring; rather, the spot size and location were fitted from the data. Hence the monitoring scheme described can only improve on the \pm 0.0005 fitting error of E-770.

We summarize the requirements for the monitoring in $\mathrm{E}-815$ as:

1. Position of primary impact point on the target with $\sigma=1 \mathrm{~mm}$.

2. Beam Angle: $\sigma_{\theta} \approx 0.25 \mathrm{mrad}$.

3. Flux to $\pm 10 \% .^{12}$

4. Beam Profile: $\sigma_{x} \approx 0.7, \sigma_{y} \approx 3 \mathrm{~mm}$, known to $10 \%$ of itself.

We will wish to measure the characteristics for each ping separately but do not expect to resolve variations within a ping. Passive devices such as BPM's are best suited for the high intensities required for a neutrino experiment, although they provide no more than the centroids. Wire SEM's measure beam profile but cannot be left in the beam. ${ }^{13}$ We will use Wire SEM's to measure the beam profile on a regular basis and if conditions change, as determined by the BPMs, additional SEM scans can be taken.

The "long" BPMs produce a stronger signal and are therefore desirable. The downstream SEM pair would have a wire spacing of $0.1 \mathrm{~mm}$. The upstream SEM pair would use standard $1 \mathrm{~mm}$ pitch.

\footnotetext{
${ }^{12}$ This is not critical for the $\sin ^{2} \theta_{W}$ analysis but could be used for other measurements and for monitoring beam conditions.

${ }^{13}$ New Wire SEM's are under development for KTeV which may be able to manage the required intensity under long-term conditions. If these are suitable, we will use them in addition to the BPM's. [9]
} 


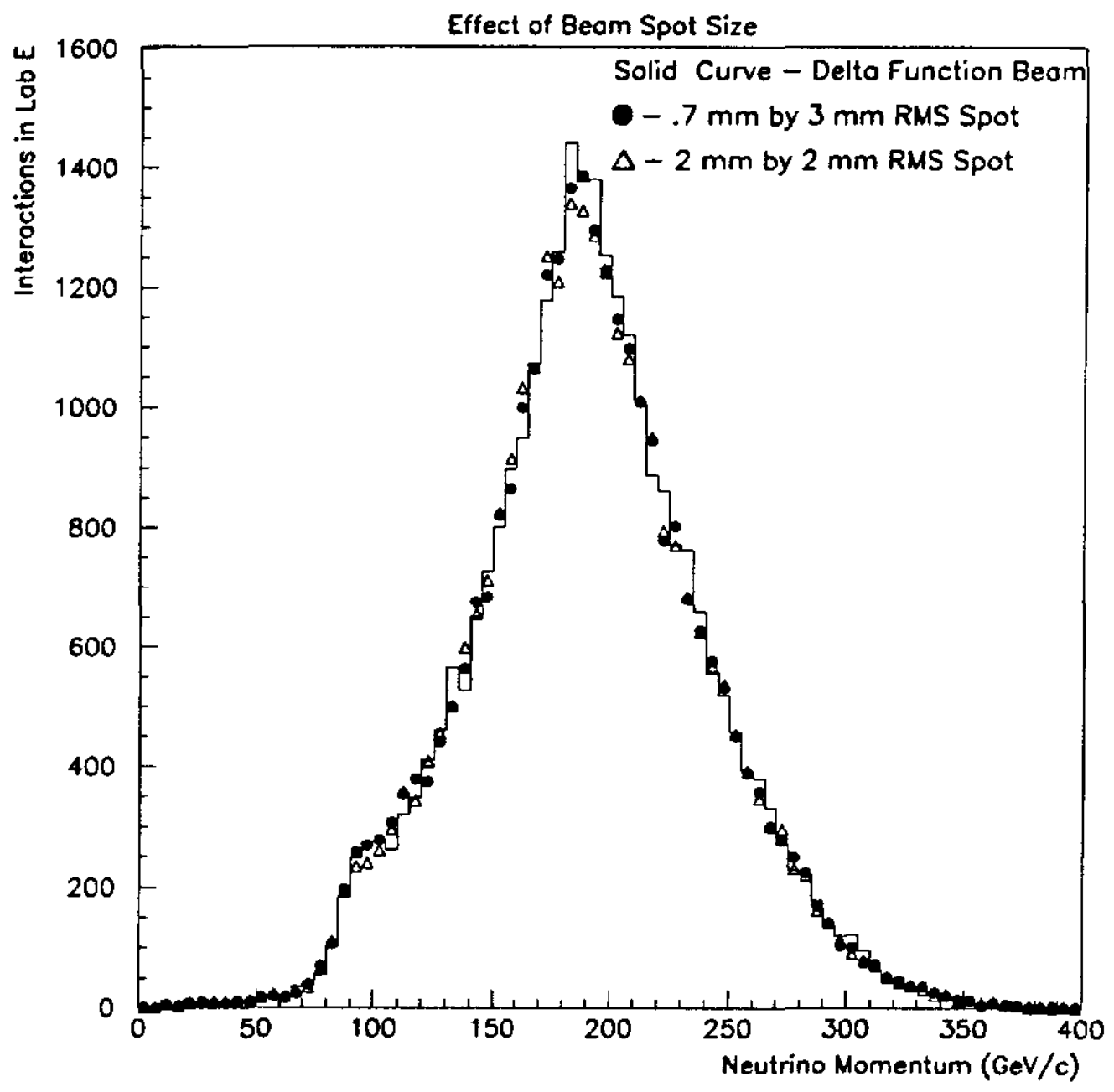

Figure 28: Effect of Beam Spot Size on the $\nu_{\mu}\left(K^{ \pm}\right)$spectrum. 


\subsection{Secondary Beam Monitoring}

Knowledge of the beam (secondary mesons) center at the $1 \mathrm{~cm}$ level should suffice. Large area SWICS $(0.8 \times 0.8 \mathrm{~m}$ with $1 \mathrm{~cm}$ pitch) will be placed in NW2. The combined remnant beam and decay secondary particles profiles at NW2 and NW4 are given in Figs. 29 and 30.

\subsection{Alignment}

The important alignment is that internal to N01. Shifted quadrupoles introduce a dipole moment which then alters the angle of the daughter mesons and the resultant position of the neutrinos in Lab $\mathrm{E}$. Accurate beam alignment is therefore invaluable in understanding the fine details of the beam. An example of the effect is shown in Fig. 31, where Q1, Q2, and $\mathrm{Q} 3$ were shifted $+30,-30$, and +30 mils off-axis. ${ }^{14}$. Hence we need the beam elements to be placed with an accuracy of $\leq 30$ mils.

Optical surveys can achieve the required 30 mil accuracy. Discussions with the survey group have led to plans for mounts on each magnet and we are investigating finding the magnetic axis by standard "floating wire" techniques.

An overall angle misalignment between the local N01 line with respect to NCenter will simply result in a displacement of the $\nu$ impact point in Lab $E$ which can be removed by small adjustments to the field of the final dipole. The final dipole will run at $\Delta \theta_{p=250 \mathrm{GeV} / \mathrm{c}}=1.75$ mrad or a transverse bend of 2.6 meters over the distance to Lab E. Hence we expect to center the neutrino distribution on the Lab $\mathrm{E}$ detector with this dipole.

\footnotetext{
${ }^{14}$ We observed a similar effect in E-770 where the third set of quadrupoles in the train were off by $\approx 30$ mils. This limitation made it difficult to understand the lower edge of the $\pi$ spectrum (where low-energy backgrounds might manifest themselves).
} 


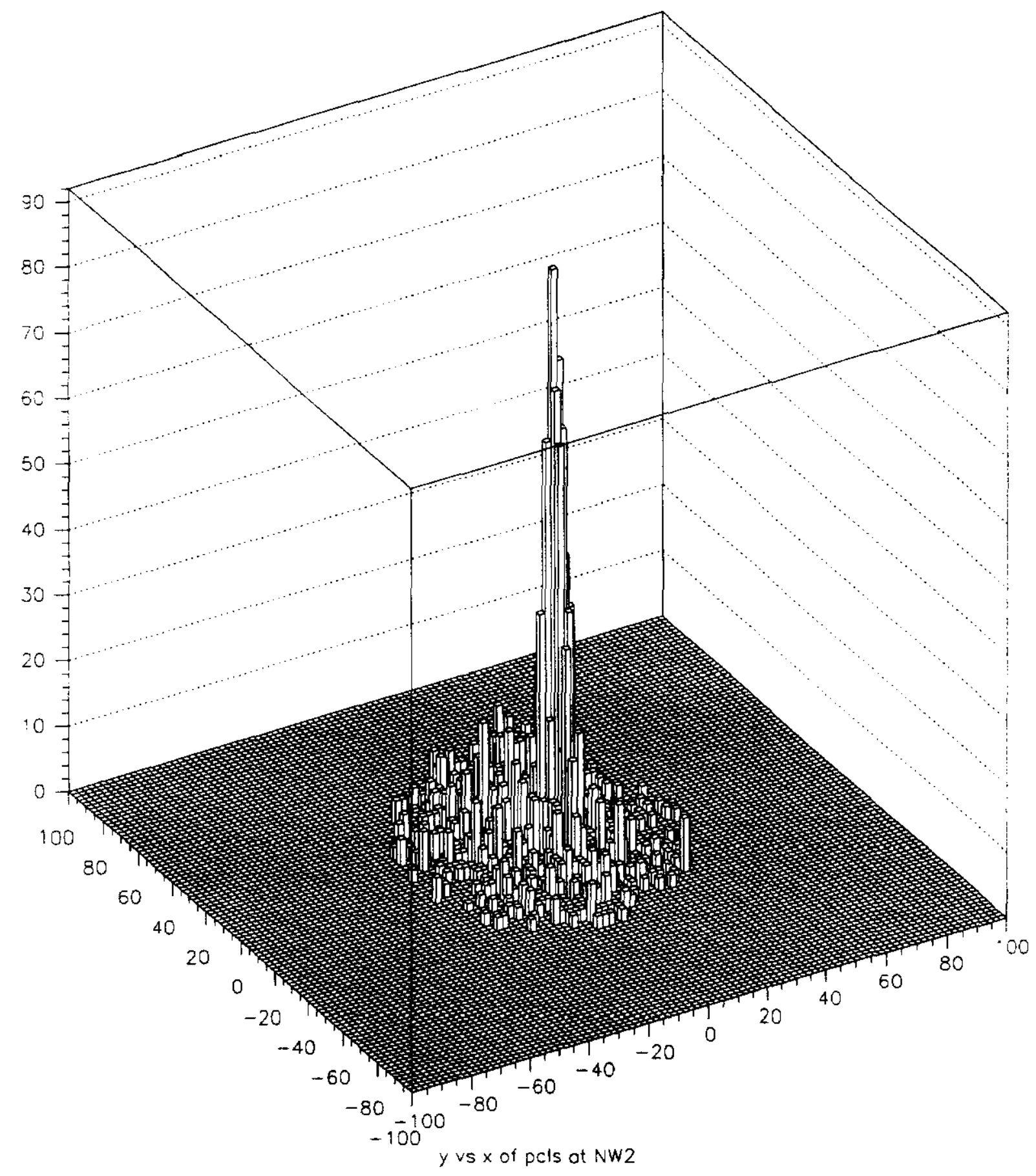

Figure 29: Beam Profile at NW2. 


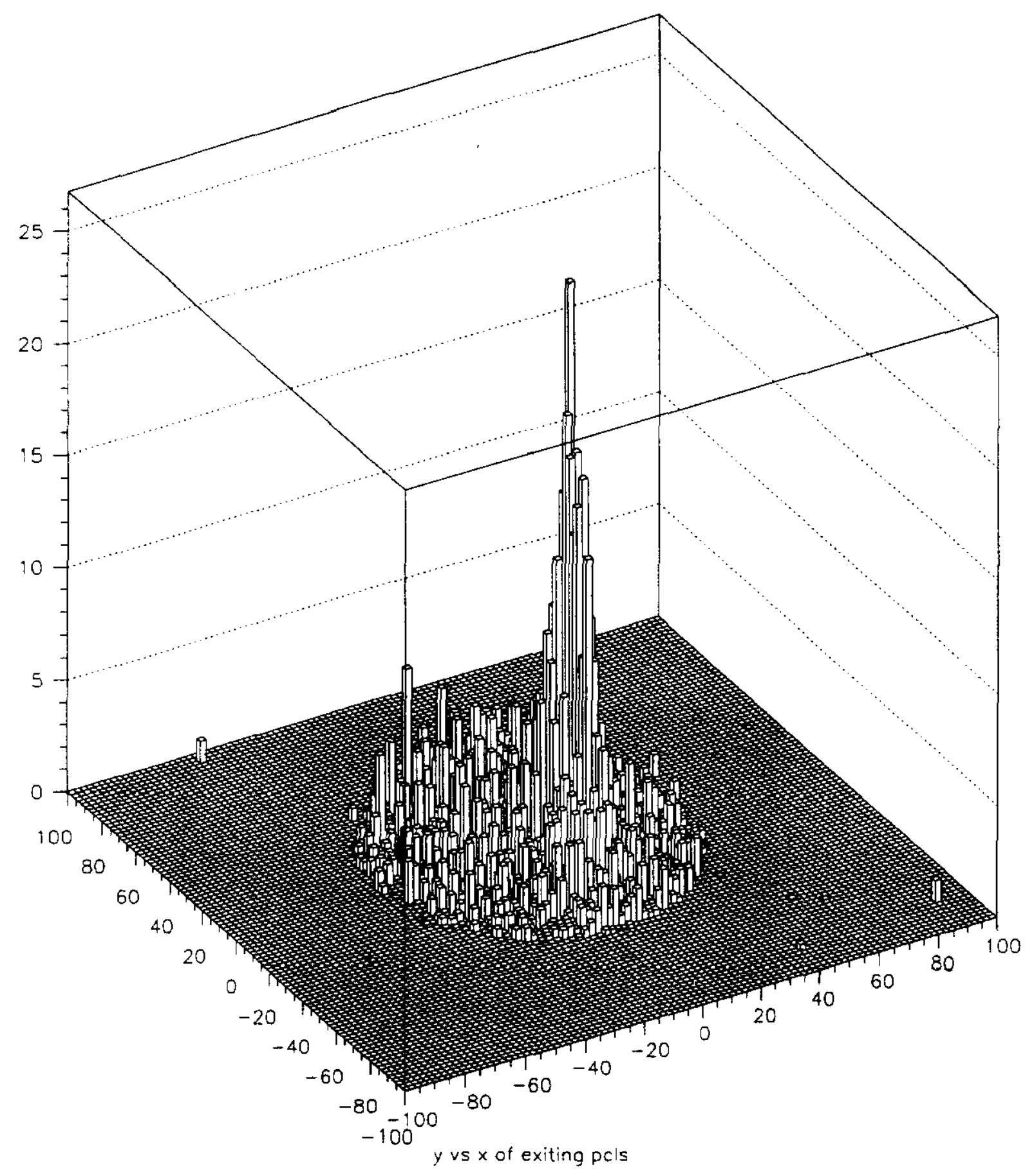

Figure 30: Beam Profile at NW4. 


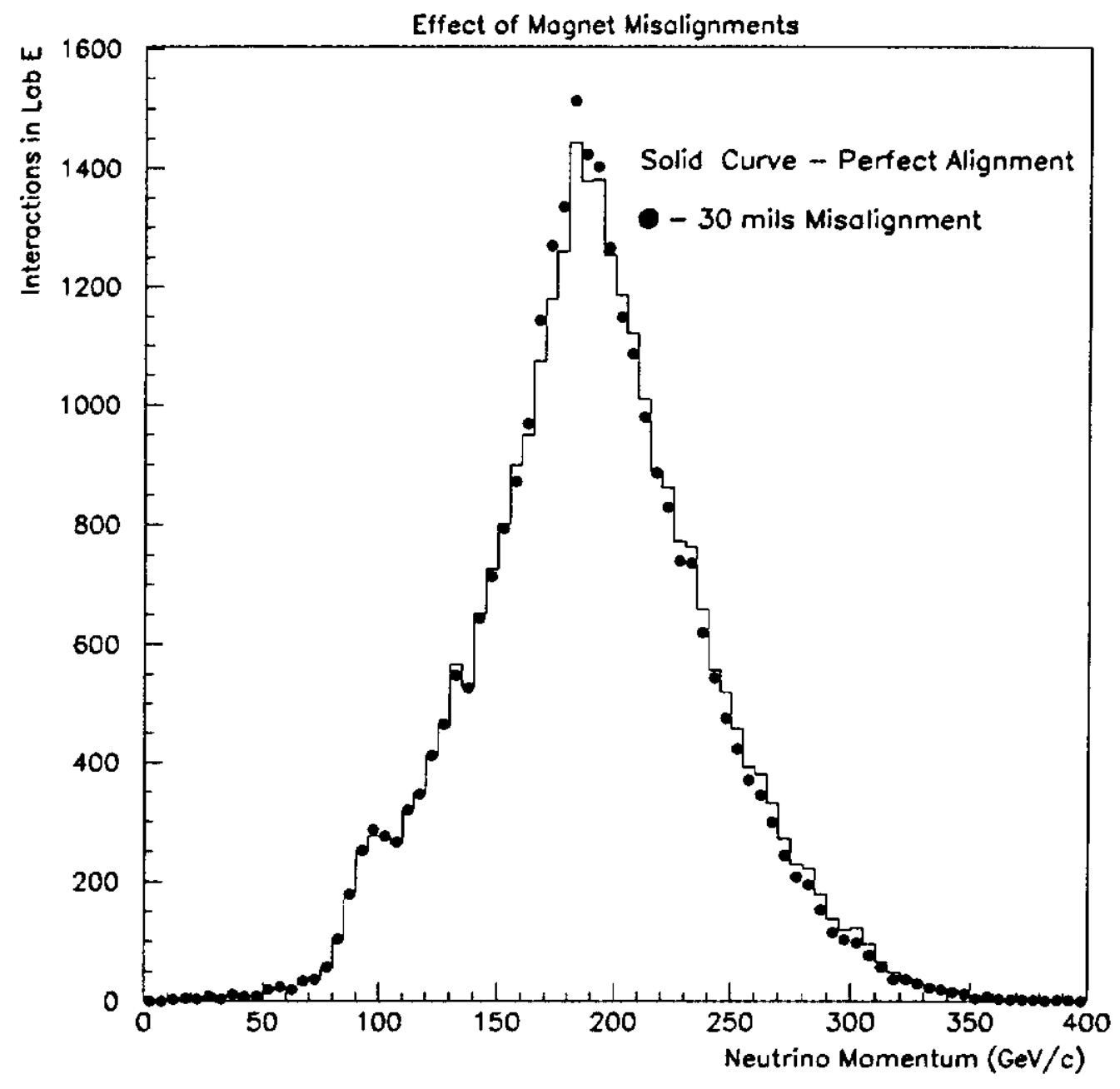

Figure 31: Effect of a displacement of quadrupoles in the SSQT. The calculation is done for Q1 displaced downwards by 30 mils, Q2 upwards by 30 mils, and Q3 downwards by 30 mils. 


\section{References}

(1) E-815 Proposal and references therein.

[2] B.J. King, XXVIIIth Recontre De Moriond, 13-20 March 1993 (to be published).

[3] A. Blondel et al., Z. Phys. C45, 361 (1990).

[4] C.H. Llewellyn Smith, Nucl. Phys. B228 (1983) 205; also see E.A. Paschos and L. Wolfenstein, Phys. Rev. D7 (1973) 91.

[5] P. Skubic et al., Phys. Rev. D18, 3115 (1978).

[6] D. Carey, The Optics of Charged Particle Beams, Harwood Press (1987).

[7] H.W. Atherton et al., Precise Measurements of Particle Production by $400 \mathrm{GeV} / \mathrm{c}$ Protons on Beryllium Targets, CERN-80-07 and A. Malensek, FN-341.

[8] E-731, priv. comm.

[9] Operations Report on E-815, 1 November 1993. 